\title{
Horizontal contraction in image domain for velocity inversion
}

\author{
Peng Shen ${ }^{1}$ and William W. Symes ${ }^{2}$
}

\begin{abstract}
A kinematically correct choice of velocity focuses subsurface offset image gathers at a zero offset. Infinitesimal warping from the current image toward its focus can be approximated by a horizontal contraction. The image residual can then be taken as the difference between the warped and the original image to account for the velocity error. Leastsquares fitting of the effect of a velocity perturbation to this image-warping perturbation produces a tomographic velocity update. We evaluated a warping scheme based on the radial image derivative in the subsurface offset. We found that the corresponding gradient was free of the diffraction edge effect. We further enhanced the efficiency of the velocity update procedure via the use of a diagonal Hessian approximation. We developed a sequence of synthetic examples leading to a salt body inversion. The limitation of the method was argued and analyzed in the presence of refracting structures.
\end{abstract}

\section{INTRODUCTION}

For classical full-waveform inversion, the observed data are naturally treated as a reference. The difference between the synthetic and the observed data (data residual) drives velocity updating through minimization of its mean square (Tarantola and Vallette, 1982; Pratt et al., 1998). Wave-equation reflection tomography is often posed in the image domain, where there is no natural reference image that can be used for comparison. Sava (2000) proposes a method to obtain a reference image via Stolt residual migration based on the information generated in the current migration. Differential semblance (DS) reflection tomography defines the residual by scaling the image by offset (Shen and Symes, 2008). Fei and Williamson
(2010) report a DS-type image residual using an infinitesimal offset contraction, whereas Albertin (2011) explicitly uses two infinitesimally separated images to produce an image residual aided with a local re- and demigration using one-way propagators. It is not clear how to extend Albertin's algorithm into general propagators because the scheme is closely tied to the double-square-root formulation.

Waveform tomography maps the image residual to a search direction in the velocity model space. For DS and similar algorithms, the search direction is (or is closely related to) the gradient of an objective function. The various choices of residual definition are not equivalent: In particular, reflector truncations and other singularities lead to oscillations in the DS gradient (so-called gradient artifacts [Fei and Williamson, 2010]), which lead in turn to slow convergence and to the need for significant smoothing on the velocity update. Image residuals obtained as differences between infinitesimally separated images appear to lead to velocity updates, which avoid these artifacts. These updates are, on the other hand, not gradients of objective functions.

All waveform-tomographic updates, including DS, may be viewed as least-squares projections of image updates into velocity model space. The rule for forming image updates may be viewed as a vector field (differential equation) on image space, limit points of which are focused (physical) images. Velocity estimation amounts to solving the projected differential equation in model space by a marching method.

This paper describes a warping method, named horizontal warping, based on the radial offset derivative, that generates infinitesimally improved images from current images. We demonstrate that the velocity update from the corresponding image residual is artifact free. We note that this velocity update is not the gradient of an objective function. Therefore, conventional quasi-Newton's methods generally used in the optimization of global objective functions are not usable. The key to efficiency is fast computation of the projection into model space; we introduce an approximate Hessian inverse, which renders this projection practical at industry scale.

\footnotetext{
Manuscript received by the Editor 2 June 2014; revised manuscript received 17 October 2014; published online 15 May 2015 ; corrected version published online 15 May 2015.

${ }^{1}$ Fomerly Shell International Exploration and Production; presently Chevron Corporation, Houston, Texas, USA. E-mail: peng.shen@chevron.com.

${ }^{2}$ Rice University, Department of Computational and Applied Mathematics, Houston, Texas, USA. E-mail: symes@caam.rice.edu.

(C) 2015 Society of Exploration Geophysicists. All rights reserved.
} 


\section{METHOD}

A 2D subsurface offset image gather for layered reflections (horizontal or tilted - it makes no difference) shows a shape similar to that depicted in Figure 1a. Depending on whether the migration velocity pertaining to the event is greater than or smaller than the true velocity, the image in the subsurface offset, denoted by $h$, curves upward or downward, respectively. In both cases, the events have a curvature direction along which an infinitesimal shift from the original image makes the final image slightly more focused. The image corresponding to the correct velocity is always on the $h=0$ (zero-offset) axis represented by the solid circle in Figure 1, near the envelope of the normal line to the initial image. Motion or warping by inward curvature is difficult to implement. However, for the cases delineated in Figure 1, a good approximation can be obtained in $h \neq 0$ by ignoring the vertical shift. The vertical shift is most evidently seen as the vertical separation in the zerooffset events represented by the dashed and solid curves in Figure 1a. As shown in Figure 1b, a horizontal contraction represented by the dashed curves well approximates the inward warping except for points at a zero offset. Let $\tilde{I}$ denote the infinitesimally warped image from the current image $I$ by a small amount $\Delta h$, where $\Delta h$ is an increment of horizontal offset along the radial direction. Let $x$ be the image point and $h$ be the horizontal subsurface offset; then, for $h \in R$,
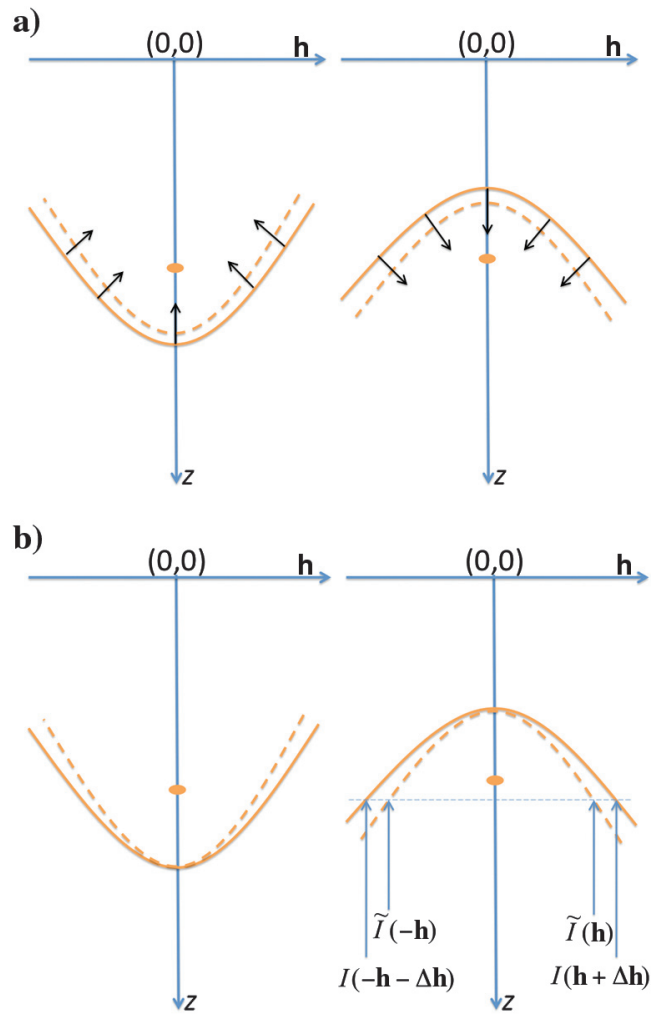

Figure 1. Schematic illustration of an image in the subsurface offset. (a) Events obtained with a faster or slower velocity curve up (left) or down (right), respectively. The black arrows indicate the warping direction. The solid and the dashed curves represent the current and the infinitesimally warped image $I$ and $\tilde{I}$, respectively. The orange solid circle represents the focus point of the event. (b) Horizontal contraction as an approximation to the total warping.

$$
\tilde{I}(x, h)=\left\{\begin{array}{cc}
I(x, h+\Delta h) & h>0 \\
I(x, h) & h=0 \\
I(x, h-\Delta h) & h<0
\end{array}\right.
$$

We regard $\tilde{I}$ as an infinitesimally improved image, moved slightly toward the true (focused) image. The difference between $\tilde{I}$ and $I$ is much less than a quarter-wavelength in the band limit and can be expressed via the radial derivative $\partial_{|h|}$ :

$$
\mathrm{DI}_{\mathrm{RAD}}=\partial_{|h|} I \simeq \frac{\tilde{I}-I}{\Delta h},
$$

where

$$
\partial_{|h|}= \begin{cases}\hat{h} \cdot \nabla_{h} & h \neq 0, \\ 0 & h=0,\end{cases}
$$

where $\hat{h}$ is the unit horizontal subsurface offset vector, $|h|$ is the radius or length of the vector $h$, and $\nabla_{h}$ is the gradient operator in $h$. Because of the bandlimitedness of seismic data, the image migrated from data is practically always differentiable in offset. Equations 2 and 3 are also correct as written in 3D (for the 2D offset vector $h$ ).

Although the warping formula (equation 2) drives the image toward focus, it is not the result of migrating the same data with a different velocity. Therefore, we project the image difference expressed in equation 2 into velocity space by solving the equation,

$$
\left(\frac{\partial I}{\partial c}\right) \delta c=\delta I_{\mathrm{RAD}},
$$

for a velocity update $\delta c$, where $\delta I_{\mathrm{RAD}}=\mathrm{DI}_{\mathrm{RAD}} \Delta h$. Because equation 4 is very unlikely to have a literal solution, we solve it in the least-squares sense; that is,

$$
\delta c=H^{-1} g,
$$

in which

$$
H=\left(\frac{\partial I}{\partial c}\right)^{*} K\left(\frac{\partial I}{\partial c}\right)
$$

is the Hessian of the least-squares objective

$$
J[\delta c]=\frac{1}{2}\left\|L\left(\left(\frac{\partial I}{\partial c}\right) \delta c-\delta I_{\mathrm{RAD}}\right)\right\|^{2},
$$

and

$$
g=\left(\frac{\partial I}{\partial c}\right)^{*} K \delta I_{\mathrm{RAD}}
$$

is its gradient against $\delta c$. Here, $K=L^{*} L$ and $L$ is a suitable preconditioning operator in image space. An example of $L$ can be the automatic gain control (AGC) operator whose purpose is to balance the image amplitude.

Rule 5 produces a velocity update for any choice of image residual $\delta I$. For example, Fei and Williamson (2010) suggest in 1D offset (compared with their original equation, a $\operatorname{sgn}(h)$ is introduced to make the equation valid for $h<0$ and $h \geq 0$ ): 


$$
\begin{gathered}
\mathrm{DI}_{\mathrm{FW}}=\operatorname{sgn}(h) h^{2} \frac{\partial I}{\partial h}, \\
\mathrm{DI}_{\mathrm{EXP}}=h \cdot \nabla_{h} I,
\end{gathered}
$$

the exponential contractive image residual (notice that the form $I\left(e^{t} h\right)$ satisfies $d I / d t=h \cdot \nabla_{h} I$ and the contraction in space is exponentially scaled). Pursuing for the radial symmetry of image in $h$ of multidimensions, the three image residuals are subject to one expression:

$$
\mathrm{DI}=X_{I} I
$$

where

$$
X_{I}=|h|^{n} \partial_{|h|},
$$

for $n=0,1,2$, corresponds to $\mathrm{DI}_{\mathrm{RAD}}, \mathrm{DI}_{\mathrm{EXP}}$, and $\mathrm{DI}_{\mathrm{FW}}$, respectively. We shall call expressions 12 , the horizontal contraction. At $n=0$, the contraction reduces to the radial derivative $\partial_{|h|}$, which is of unit strength, whereas at $n=1,2$, the contraction can be viewed as the radial derivative scaled by different amplitude factors. The expressions 12 subject to $n=0,1,2$ are of special interest for their closure under the Lie brackets (Olver, 1993).

Alternatively, "classical" differential semblance, such as, for example, in Shen and Symes (2008), uses

$$
\mathrm{DI}_{\mathrm{DS}}=A^{*} A I
$$

in which $A$ is any linear operator on the image space that annihilates focused images - multiplication by $|h|$ is the conventional choice. The residual defined in equation 13 is the image gradient of the objective function at homogeneous of degree two in $I$ via a scalar product:

$$
J=\frac{1}{2}\langle I, A I\rangle
$$

None of $\mathrm{DI}_{\mathrm{RAD}}, \mathrm{DI}_{\mathrm{FW}}$, or $\mathrm{DI}_{\mathrm{EXP}}$ is the image gradient of the objective function in the form of equation 14, and indeed the operators $X_{I}$ involved in these latter residual formulas are skew-symmetric as opposed to $A^{*} A$, which are symmetric.

For industry-scale problems, iterative schemes for inverting the Hessian $H$ often become prohibitively expensive. Fei and Williamson (2010) use the approximation $H=$ identity operator. An economical and much more accurate approximation to equation 5 stems from the observation that

$$
\tilde{H}_{d}=\left(\frac{\partial I}{\partial c}\right)^{*} K\left(\frac{\partial I}{\partial c}\right) 1_{c}
$$

approximates the diagonal of the Hessian, where $1_{c}$ is a constant velocity perturbation field that has unit value everywhere. Jointly influenced by the data and the current velocity, the diagonal of the Hessian $H_{d}$ has a physical meaning of the image-strength weighted sum of ray coverage. We then write the approximate solution to equation 4 as

$$
\tilde{\delta c}(x)=\frac{g(x)}{\tilde{H}_{d}(x)},
$$

in which the inverse Hessian is replaced by a pointwise division of the gradient $g$ by the approximate diagonal of the Hessian at every $x$. Operators $(\partial I / \partial c)$ and $(\partial I / \partial c)^{*}$, as an adjoint pair, can be formulated in wave-equation settings as described in Shen (2012). For completeness, we reformulate and reinterpret them in Appendix A.

\section{VELOCITY INVERSION VIEWED AS A DYNAMICAL SYSTEM}

The velocity in a "migration velocity analysis" can be viewed as an implicit function of image that is further viewed as a function of "time" $t$ or the evolution parameter, over which the system velocity evolves. The orbit that the velocity $c(t)$ travels during inversion is a curve, and is almost a ray, except that it has not been associated with a symplectic structure. Nevertheless, we can always treat it as the solution to an ordinary differential system. Let the image under some preconditioning $L$ be represented as $I_{l}=L I$, then we have

$$
\frac{\partial I_{l}}{\partial c}=\frac{\partial I_{l}}{\partial I} \frac{\partial I}{\partial c}=L \frac{\partial I}{\partial c}
$$

total differential of velocity $c\left(I_{l}(I(t))\right)$ with respect to $t$ can be written in a chain rule as

$$
\frac{d c}{d t}=\frac{\partial c}{\partial I_{l}} \frac{\partial I_{l}}{\partial I} \frac{d I}{\partial t}=\frac{\partial c}{\partial I_{l}} L \frac{d I}{d t}
$$

Writing $\frac{\partial c}{\partial I_{l}}$ as a symbolic inverse to $\frac{\partial I_{l}}{\partial c}$, equation 18 becomes

$$
\frac{d c}{d t}=\left(\frac{\partial I_{l}}{\partial c}\right)^{-1} L \frac{d I}{d t} .
$$

The inverse $\left(\frac{\partial I_{l}}{\partial c}\right)^{-1}$ can be interpreted as the generalized inverse, i.e.,

$$
\left(\frac{\partial I_{l}}{\partial c}\right)^{-1}=\left(\left(\frac{\partial I_{l}}{\partial c}\right)^{*}\left(\frac{\partial I_{l}}{\partial c}\right)\right)^{-1}\left(\frac{\partial I_{l}}{\partial c}\right)^{*} .
$$

Substitute equation 20 into equation 19 and using equation 17, we obtain

$$
\frac{d c}{d t}=\left[\left(\frac{\partial I}{\partial c}\right)^{*} K\left(\frac{\partial I}{\partial c}\right)\right]^{-1}\left\{\left(\frac{\partial I}{\partial c}\right)^{*} K \frac{d I}{d t}\right\} .
$$

Again $K=L^{*} L$ is the same setting following equation (8). Equation 21 is essentially the same as equation 5 , but rederived form a dynamical system argument. In fact, the term contained in the curled brackets is the gradient and the term in the square bracket is the Hessian. Treating velocity as a solution to the dynamical system, we can solve equation 21 by a standard Euler's method (Kincaid and Cheney, 1996). Additional details of the Euler updates including regularizations in image as well as velocity are treated and explained in Appendix B. The construction of the dynamical system above reconfirms the procedure of least-squares projection described in the method section. They lead to the essential contention of this paper: A rule for constructing an image residual as 


$$
\delta I=\frac{d I}{d t} \delta t
$$

gives rise to a rule for a velocity update $\delta c$ via least-squares projection from image space to velocity space.

If we cast the $d I / d t$ in equation 22 under the evolution system in image as

$$
\frac{d I}{d t}=X_{I} I
$$

where $X_{I}$ is an infinitesimal generator defined by equation 12 , its solution can be derived

$$
I_{t+\delta t}(h)=e^{\delta t X_{I}} I_{t}(h)=I_{t}\left(e^{\delta t X_{I}} h\right),
$$

where $I_{t}(h)$ is the image viewed as a function of $h$ but realized at $t$. The last "=" sign assumes the convergence of the Taylor expansion. For the three choices given by equation 12 subject to $n=0,1,2$, the above equation renders $I_{t+\delta t}(h)=I_{t}(h+\delta t \cdot \hat{h}), I_{t+\delta t}(h)=$ $I_{t}\left(e^{\delta t} h\right)$, and (for $\left.0<\delta t<1 /|h|\right) I_{t+\delta t}(h)=I_{t}\left(\frac{h}{1-\delta t|h|}\right)$, respectively. All of the above are contracted in the radial direction for sufficiently small $\delta t>0$ along the flow in image characterized by the one parameter group of transformation $\phi_{I}(\delta t)=\exp \left(\delta t X_{I}\right)$ generated via $X_{I}$.

On the other hand, we can associate a coordinate system to velocity by representing the velocity in the Cartesian grid. The velocity value at the grid is viewed as the coordinate value. In this way, we write it as

$$
c=\left(c^{1}, c^{2}, c^{3}, c^{4}, \ldots, c^{n}\right),
$$

where the super index is the grid index, and $n$ is the total number of grids. Subject to this coordinate system, we introduce the vector field in velocity as

$$
X_{c}=\sum_{i=1}^{n} \frac{d c^{i}}{d t} \frac{\partial}{\partial c^{i}}
$$

Noticing $\partial c^{i} / \partial c^{j}=\delta_{j}^{i}$ (Kronecker delta) and the operator in velocity domain $d / d t=X_{c}$, equation 21 can be expressed in the form:

$$
c(t+\delta t)=\exp \left(\delta t X_{c}\right) c(t) .
$$

We have written the velocity as a local Lie group via the exponential map. The one parameter group of transformation $\phi_{c}(\delta t)=\exp \left(\delta t X_{c}\right)$ is generated by the vector field $X_{c}$ which is converted from a vector field in image $X_{I}$. We interpret the local Lie group in velocity as follows. For every infinitesimal generator $X_{I}$ in image, there corresponds an infinitesimal generator $X_{c}$ in velocity. Equation 21 suggests that these two vector fields relate to each other via the tangent map $\frac{\partial c}{\partial l}$, which is implemented as the generalized inverse. The infinitesimal generator in image generates a flow that brings the image into focus. In the same time, the flow in image is converted to, and therefore determines a flow $\phi_{c}$ in velocity that brings the velocity to the correct one. A physical evolution of velocity that brings the velocity to the true induces the flow in image that contracts the image to focus. Inversely, velocity inversions can be triggered by contractive flows in image, among which the horizontal contraction becomes the simplest.

\section{LIMITATIONS OF HORIZONTAL CONTRACTION}

The image-domain flow that focuses the image as described by equation 23, which we shall call horizontal warping, is generated by the horizontal contractive infinitesimal generator $X_{I}=|h|^{n} \partial_{|h|}$. This image-domain flow does not necessarily reenact the responses of an image to a physical evolution of velocity. An obvious example is when as the velocity approaches to the true, the image in offset shifts vertically, a motion that is entirely missed in horizontal warping. The vertical shift is most clearly seen as the vertical arrow sitting at the crossing point of the event and the zero-offset axis, shown in Figure 1a. However, the least-squares projection in an infinitesimal step invoked by horizontal warping can well fill in what corresponds to the missing vertical shift. In other words, the tangent map $\partial c / \partial I$ implemented as the generalized inverse produces a velocity update that feeds back to the image through migration, so that the required vertical motion is reinserted. This seems to be valid for velocities that are void of refracting structures as demonstrated by examples in later sections.

On the other hand, when a refracting velocity is present, the evolutional path an image takes may be negatively projected onto the path that the horizontal warping prescribes. To demonstrate this, we generate Born data according to

$$
\begin{aligned}
p\left(x_{r}, t \mid x_{s}\right)= & \int d x d \tau d x_{r} G\left(x_{r}, t \mid x, \tau\right) \frac{2 \delta c(x)}{c^{3}(x)} \partial_{\tau}^{2} G\left(x, \tau \mid x_{s}, t_{r}\right) \\
& \times f\left(x_{s}, t_{s}\right),
\end{aligned}
$$

where $G\left(x, \tau \mid x_{s}, t_{r}\right)$ is a Green's function evaluated at location $x$ and time $\tau$ due to the spike located at $x_{s}$ time $t_{r}$; the Born data $p\left(x_{r}, t \mid x_{s}\right)$ are received at $x_{r}$ at time $t$; the source $f$ has a peak frequency at $13 \mathrm{~Hz}$ with an increment of $40 \mathrm{~m}$ in $x_{s}$; and $\delta c$ is made of nine horizontal singular reflectors embedded in a $2 \mathrm{D}$ velocity model $c$, which consists of a background velocity at $2000 \mathrm{~m} / \mathrm{s}$ and a low-velocity lens centered with $1600 \mathrm{~m} / \mathrm{s}$ (Figure 2a). The acquisition is split spread with maximum surface offset of $1500 \mathrm{~m}$. The shot gather directly above the lens shows clear triplication (Figure $2 b$ ). The gradient obtained with constant velocity at $2000 \mathrm{~m} / \mathrm{s}$ presents a sign flip on the bottom half in the model space. The diagonal Hessian correction only modifies the amplitude but keeps the sign unchanged. Lens data with different refracting strength repeat the same gradient pattern as shown in Figure 2c. We extract the offset gather through the middle of the model. In Figure 2d, the offset runs from -150 to $+150 \mathrm{~m}$ horizontally. Shown in Figure 2e (right) is a plot schematically representing the offset gather in Figure $2 \mathrm{~d}$. The data record the multivaluedness of traveltimes that manifests itself as a set of crossing events near the zero subsurface offset. This is a phenomenon quite different from the layered case as demonstrated in Figure 2e (left). In Figure 2e (right), the vertical shift for the bottom event required by the physical velocity update is contradicted by the horizontal contraction. We hypothesize that the correct total warping to produce an infinitesimally improved image is a perturbation toward the direction that increases the image curvature, while keeping the image gather centered at a zero offset. We see that in the layered case, the direction of warping by horizontal contraction (blue arrow) forms a less-than- $90^{\circ}$ angle with the direction of total warping (black arrow). For the case of a low-velocity lens, this angle is greater than $90^{\circ}$ for deeper images, where the multivaluedness breaks the horizontal smoothness of the image at a 
zero offset. In other words, the evolution of image to focus does not necessarily follow a horizontal contraction path; the horizontal contraction is not a good approximation of the total warping if it cannot increase the resulting image curvature.

\section{EXAMPLES}

In all of the following examples, the inversion complexity increases sequentially. However, we try to avoid the lensing structures. Example 1 shows that the gradient induced by horizontal warping alone is smooth, in which no Hessian correction is needed. When there is no global objective function available for the velocity inversion, a direct implementation of the inverse Hessian becomes important. Example 2 explains the use of the inverse Hessian correction through the diagonal approximation. In example 3 , we impose regularizations in the image domain when the image amplitude varies dramatically, a situation often encountered when the salt body is embedded in sediments. In example 4, to invert for the salt body it is required to impose regularizations not only in the image but also in velocity. We also give an analysis of stack power in comparison with image residuals generated by skew-symmetric operators.

\section{Example 1: Smooth gradient}

We first demonstrate that the image residual induced by horizontal infinitesimal contraction does not produce gradient artifacts. The synthetic data are made from an acoustic full-waveform simulation (Shen, 2012) with reflections generated by positive velocity perturbations designed to have sharp edges. Figure 3 a shows the image of reflectors obtained at the correct velocity. Using conventional DS image residuals, such as $\delta I=h^{2} I$, we obtain a gradient according to equation 8 (with $K$ set equal to the identity operator) as shown in Figure $3 \mathrm{~b}$ and $3 \mathrm{c}$ for slower and faster velocities, respectively. The white color is negative, and black is positive. Compared with one-way propagators, the reverse-time propagation kernel actually produces stronger and more complicated diffraction edge effects in the gradients. Shown in Figure $3 \mathrm{~d}$ and $3 \mathrm{e}$ are gradients obtained with slower and faster velocities, respectively, using image residuals $\delta I=-\partial_{|h|} I$. Clearly, with the same gradient operator $(\partial I / \partial c)^{*}$, the image residual induced by the radial derivative produces gradients (Figure $3 \mathrm{~d}$ and $3 \mathrm{e}$ ) not only correct in sign but also free of the oscillatory edge artifacts, compared with those obtained by conventional DS (Figure $3 \mathrm{~b}$ and $3 \mathrm{c}$ ).

The horizontal contractive infinitesimal generator produces a $90^{\circ}$ phase shift toward the focus in $h$. Suppose the signal is of an ideal broadband, then at the correct velocity, the infinitesimal generators $|h|^{n} \partial_{|h|}$ assign the image at $h=0$ to zero. Therefore, there is no velocity update produced. If the signal is bandlimited, there will be a neighborhood of correct velocity subject to which the current image and the true image are not uniformly separated by $90^{\circ}$ in-phase under the bandwidth given by the signal. To this situation, the gradient may become oscillatory. We report that, for a homogeneous true background velocity at $2000 \mathrm{~m} / \mathrm{s}$ and a source wavelet of peak frequency at $15 \mathrm{~Hz}$ (maximum frequency $\sim 30 \mathrm{~Hz}$, so the smallest quarter-wavelength is $66 \mathrm{~m}$ ), a homogeneous velocity of 1950 or $2050 \mathrm{~m} / \mathrm{s}$ would still produce a smooth gradient without generating an oscillating pattern. This is quite consistent with the analysis given above.

Figure $3 \mathrm{~d}$ and $3 \mathrm{e}$ shows a vertically piecewise smooth pattern with an increasing amplitude from deep to shallow. This pattern coincides with the overlay of illuminations received by each image. The gradient itself does not correspond to a physical velocity update because of the incorrect amplitude variations mentioned above. Assuming that a global objective function is available, a typical quasiNewton method derives the velocity update by indirectly building the inverse Hessian through iterations. We will show in the next example that a direct approximation of the inverse Hessian can be achieved and subsequently applied to the gradient, so that with a)

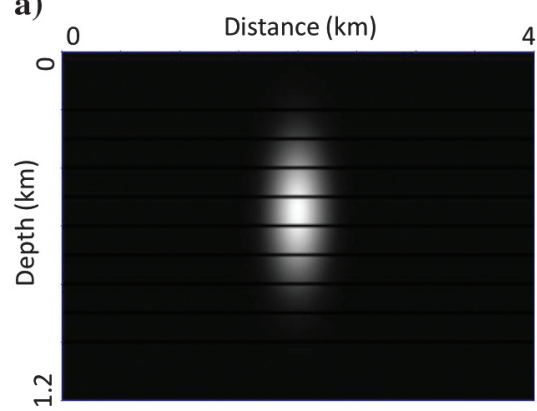

b)

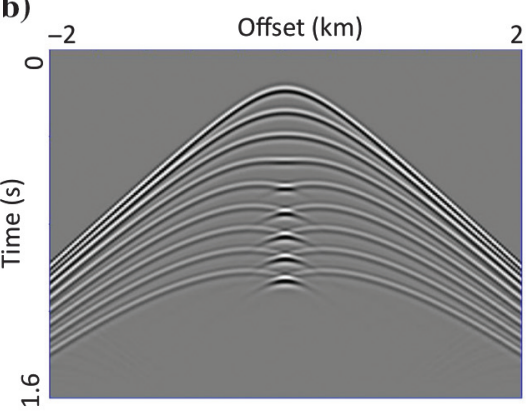

c)

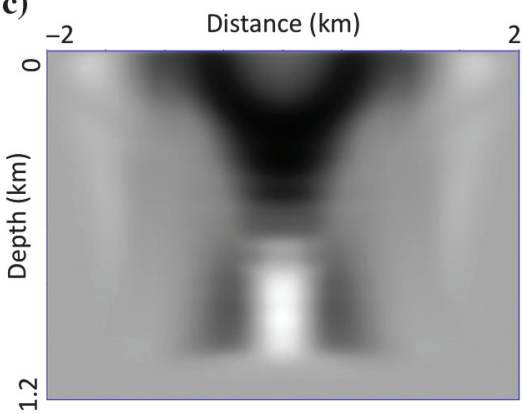

d)

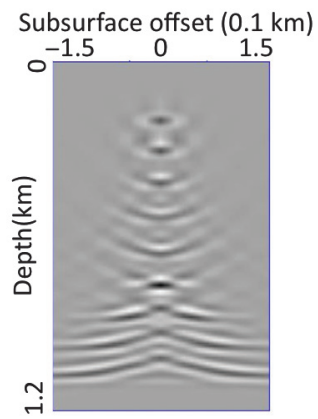

e)

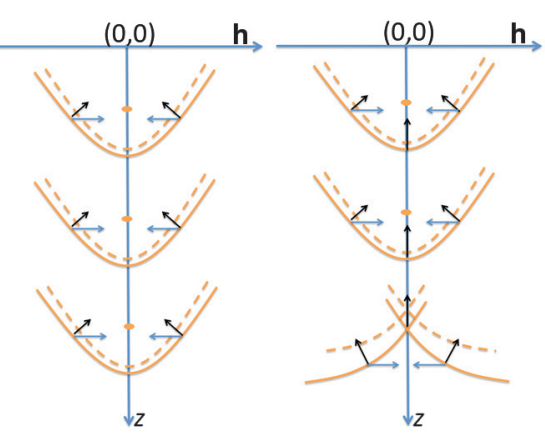

Figure 2. (a) The true lens velocity and horizontal reflectors, (b) shot gather directly above the lens, (c) the gradient obtained with constant velocity, (d) the offset gather in the middle of the model, and (e) schematic plot of the image in offset for layered media (left) and for a strong low-velocity lens (right). The blue arrows point to the direction of pullback for the horizontal contraction. 
amplitude adjustment, a physical velocity update is obtained in one iteration.

\section{Example 2: Sedimentary Born data}

The gradient (equation 8) can be viewed as a stacking of many wavepath kernels. The fact that the gradient decays in amplitude from the top of the model to the bottom of the model is primarily due to two factors. First, wavepaths overlay with a higher density in the shallow than in the deep. Second, the image illuminated with more wavepaths is naturally stronger in amplitude than otherwise, which, in turn, amplifies the shallower wavepaths. These factors are captured in the Hessian operator:

$$
H=\left(\frac{\partial I}{\partial c}\right)^{*} K\left(\frac{\partial I}{\partial c}\right)
$$

Here, the positive definite operator $K$, as described in the "Method" section following equation 8 , is an image amplitude modifier, and $H$ itself maps velocity perturbations to wavepaths subject to the influ- a)

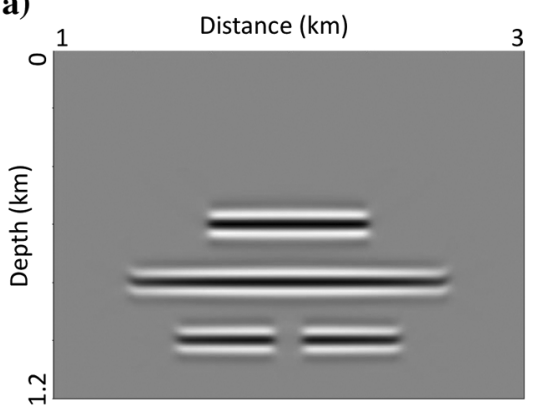

b)

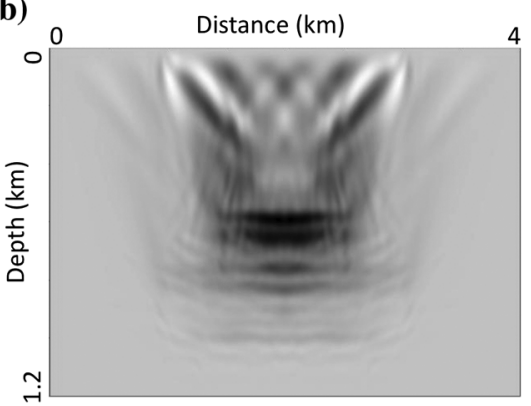

c)

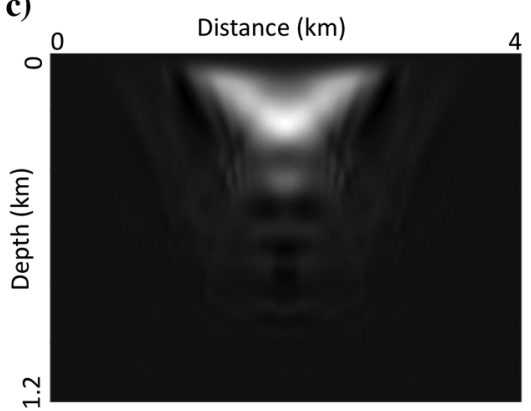

d)

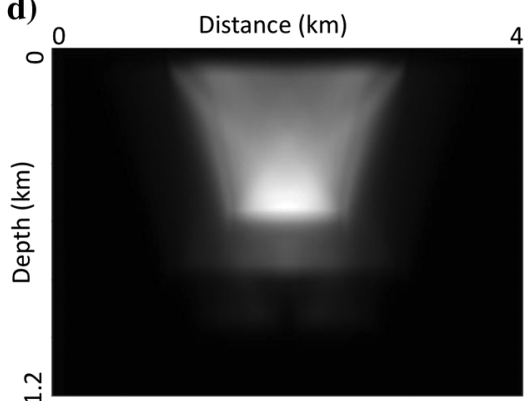

e)

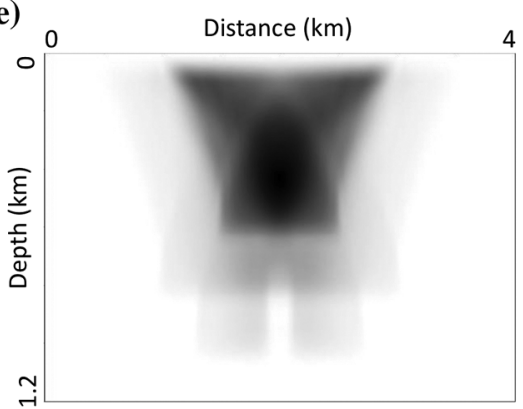

Figure 3. (a) Image with correct velocity. Panels (b-e) are gradients obtained according to equation 8 at different velocities and with different image residuals $\delta I$ : (b) slower velocity $\delta I=h^{2} I$, (c) faster velocity $\delta I=h^{2} I$, (d) slower velocity $\delta I=-\partial_{|h|} I$, and (e) faster velocity $\delta I=-\partial_{|h|} I$. ence of $K$. To illustrate the effect of the Hessian, we simulate the Born data using a time-domain acoustic propagator (equation 28). Figure 4 a shows the background velocity overlaid with velocity perturbations, a structure that is built to simulate the usual sedimentary environment. In total, 100 sources are evenly distributed across the surface of the model from 0 to $4 \mathrm{~km}$. The receiver array, consisting of 200 receivers, moves with the source at a maximum surface offset of $1500 \mathrm{~m}$, which equals the depth of the model. A singular velocity perturbation $\delta c_{i}\left(x ; x_{i}\right)$ with unit value at point $x=x_{i}$ and zero elsewhere is shown in Figure $4 \mathrm{~b}$. The Hessian response to $\delta c_{i}\left(x ; x_{i}\right)$ can be viewed as the gradient response to the image perturbation $(\partial I / \partial c)^{*} \delta I$, where $\delta I$ is induced by some velocity perturbation via $\delta I=K(\partial I / \partial c) \delta c$. In this case, assuming $K$ is identity, the application of the Hessian on $\delta c_{i}$

$$
H\left(x ; x_{i}\right)=\left(\frac{\partial I}{\partial c}\right)^{*}\left(\frac{\partial I}{\partial c}\right) \delta c_{i}\left(x ; x_{i}\right),
$$

as seen in Figure 4c, is to be localized where the singular velocity is placed, at which point the diagonal of the Hessian $H_{d}\left(x_{i}\right)=$ $H\left(x_{i}, x_{i}\right)$ is also evaluated. The localizing effect results from multiple wavepaths intersecting at the common point where the velocity perturbation is supported. For this reason, a good localization relies on a wide illumination angle from data to the subsurface point. Assuming that sufficient illumination can be provided in general, if we relocate the singular velocity perturbation to a different point, say, $x_{j}$, the Hessian $H$ subject to $\delta c_{j}$ would localize at $x_{j}$ disjointly from the focusing of $H$ subject to $\delta c_{i}$. Because the Hessian is linear and the localization of the Hessian of singular velocity perturbations is disjoint, we can superimpose singular velocity perturbations at all points, thus a constant velocity perturbation $1_{c}$, and compute its Hessian response according to equation 15 . The result evaluated at model point $x, \tilde{H}_{d}(x)$, is approximately equivalent to superpositioning $H_{d}(x)$ that are separately computed subject to each singular velocity perturbation.

The approximated diagonal of Hessian $\tilde{H}_{d}$ from equation 15) thus provides a model consistent illumination imprint. This imprint is the result of wavepath kernel stacking, which is computed in correspondence with the acquisition, imaging condition, and postmigration operation $K$. To see its effect, we first set up the initial velocity by an incorrect vertical gradient as depicted in Figure 4d. The approximated diagonal of the Hessian $\tilde{H}_{d}$ is shown in Figure 4e. The pointby-point division of the gradient (Figure 4f) by $\tilde{H}_{d}$ followed by taking a minus sign and replacing by constant values near the edges produces the search vector shown in Figure $4 \mathrm{~g}$, which is mostly homogeneous in the entire depth extent and thus is what a physical velocity update would expect. With some additional smoothing, such as a smearing at a length scale of $50 \mathrm{~m}$ with a 
B-spline (Shen and Symes, 2008) for all iterations, the Hessian-corrected gradient as an ascent direction is then fed into the inversion scheme. The inversion scheme is described in Appendix B. The velocity at iteration 20 is shown in Figure $4 \mathrm{~h}$. We point out that a direct implementation of the inverse Hessian becomes an approachable scheme for the wave-equation-based velocity inversion when the gradient is no longer associated with any global objective function.

We collect the results in Figure 5 for comparisons of various quantities before and after the inversion. Compared with the initial image as shown in Figure 5a, the image in Figure 5b agrees well with the true reflector, especially for the central part of the model. In terms of focusedness, the gathers in offset become much more focused comparing the initial (Figure 5c) and the result of iteration 20 (Figure 5d). In the same time, the gathers in the angle show significant improvement in flatness from the initial gather (Figure 5e) to the final gathers (Figure 5e) at iteration 20.

\section{Example 3: Sedimentary velocity inversion influenced by top salt}

The conventional DS-type wave-equation tomography suffers in at least two aspects in Gulf of Mexico applications. First, the irregularity of the top salt often introduces an oscillatory gradient, known as the nonsmooth gradient or gradient artifacts, which leads to difficulties for physical velocity updates. Second, the angle illumination gaps for shallow images, induced from the relatively large opening angle viewing from the subsurface point to shot intervals, introduces discontinuities in the image in angle along the moveout direction (Xu et al., 2001). The problem manifests itself in the subsurface offset as strong image amplitudes in the far offset at the correct velocity. Shen (2013) argues an approach using the focusedness of diving waves in the shallow to overrule the DS objective functions. However, it is still desirable to address the problem within the reflection regime.
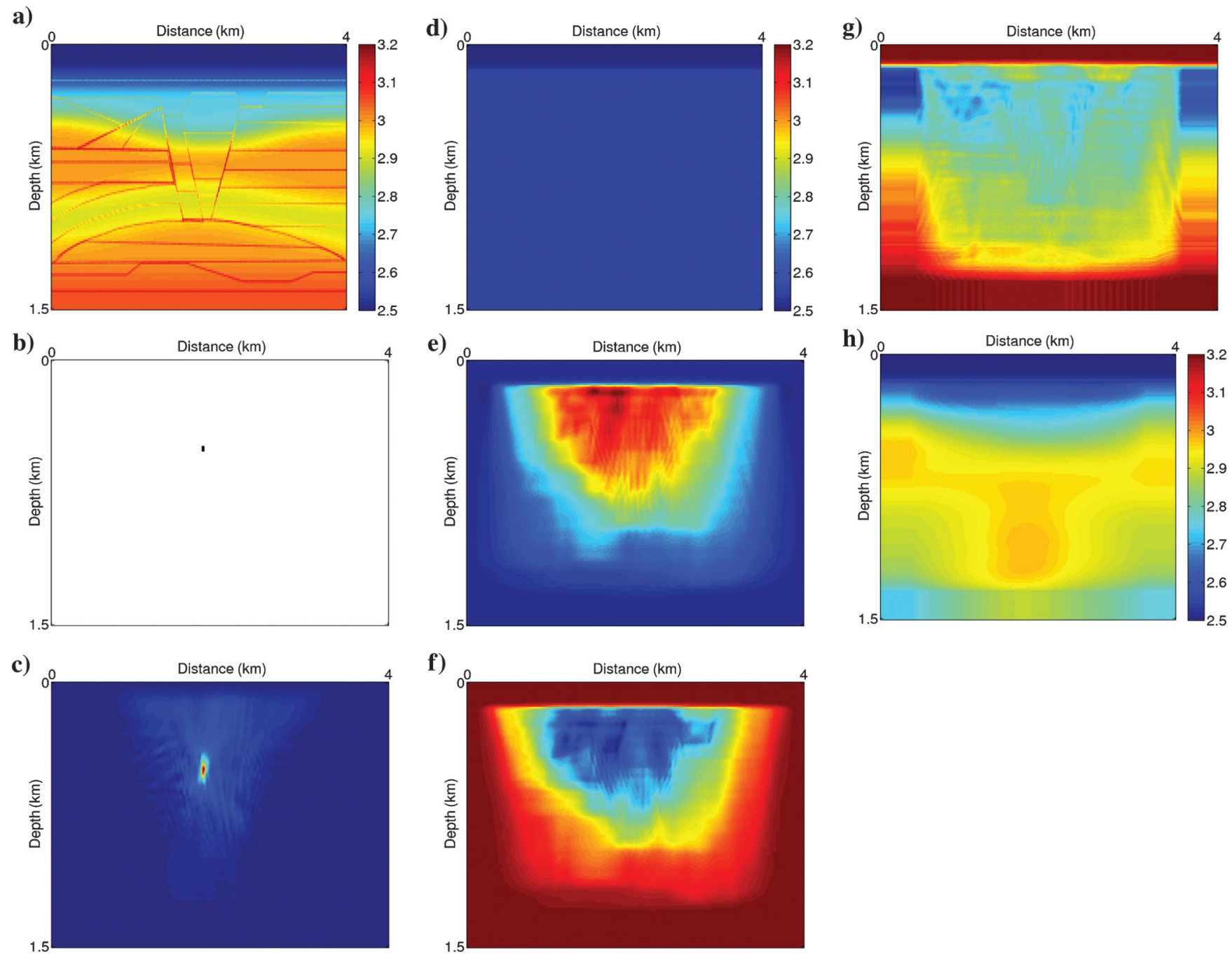

Figure 4. (a) True velocity overlaid with reflectors as velocity perturbation. (b) Singular velocity perturbation $\delta c_{s}$ that has value one represented in black and zero elsewhere represented by white. (c) Gradient response to $\delta c_{s}$, also viewed as the Hessian operator applied to $\delta c_{s}$. (d) Initial velocity that contains the correct velocity in the top layer down to $200 \mathrm{~m}$ and the incorrect constant velocity from $200 \mathrm{~m}$ to bottom. (e) Approximated diagonal of the Hessian via formula $(\partial I / \partial c)^{*} K(\partial I / \partial c) 1_{c}$ at the initial velocity for $K$ being the identity operator. (f) Gradient evaluated at the initial velocity. (g) Initial gradient divided by the initial approximated diagonal of Hessian pointwise. Panels (e-g) are muted in the water column to freeze the water velocity. (h) Velocity obtained at iteration 20. 
We full-waveform simulated the 2D acoustic synthetic data (Shen, 2012) with a rather realistic Gulf of Mexico situation, in which the above salt reflections are assumed to be relatively simple. All reflections are caused by velocity contrasts. Illustrated in Figure $6 \mathrm{a}$, the model is approximately $18 \mathrm{~km}$ wide and $6 \mathrm{~km}$ in depth. The left side top salt contains geologic irregularities. Although the right side salt is meant to be smooth on the top salt, the digitization introduces another set of irregularities. The receiver array moves along with the shotpoint at a maximum offset equal to the depth of the model. In total, 130 shots are simulated with a shot interval of $150 \mathrm{~m}$. There is a obliquity factor, implemented as $\partial / \partial z$, applied to the data to reduce the strength of the direct arrival. We can see in a typical shot gather (Figure 6b) that the amplitude of diving waves is much reduced. The image at the correct velocity is shown in Figure $6 \mathrm{c}$. With an overall slower starting velocity, the gradient of conventional DS as shown in Figure 6d contains oscillations originated from the discontinuities of the top-salt image. The gradient from horizontal warping as shown in Figure 6e, however, is still smooth with the correct sign over a large space across the model.

In these data, the top-salt reflections dominate the amplitude variations of the image as a function of velocity. It occurs that there exists many suitable above-salt sedimentary velocities that would equally focus the top-salt image. The problem is that these velocities will defocus the images in the sediment region to various degrees. To bring images of all depths to weight equally for the inversion, we calibrate the operator $K$ as described in the "Method" section. A characteristic difference between this example and the previous one is that $K$ cannot be simplified to identity. The operator $K$ can be the AGC operator. Typically, a set of coefficients is derived based on the zero subsurface offset image volume for each iteration.
These coefficients are then applied to the image in all offsets as a constant function in the offset and a variable function in spatial coordinates. In a usual DS-type algorithm driven by a global objective function, one needs to either fix the AGC coefficients for all iterations or differentiate them against the velocity. The inversion scheme we use, described in Appendix B, tremendously simplifies this requirement because $K$ is allowed to vary from iteration to iteration and the convergence is guaranteed by Euler's method instead of an accurate adjoint state evaluation of the gradient as usual. In other words, there is no need to differentiate the postmigration image manipulation against the velocity nor to keep such manipulations fixed. The operator $K$ can be any physically meaningful operator, differentiable or not differentiable, that helps to improve the condition of the inverse problem. In this work, we do ask $K$ to be self-adjoint according to the derivation posed in the previous sections.

The initial velocity is shown in Figure 7a, and the velocity inversion at iteration 9 is shown in Figure $7 \mathrm{~b}$. The above-salt section closely represents the true velocity. We can see significant improvements in the image at iteration 9 (Figure 7d) compared with the initial image (Figure 7c). In terms of the focusedness of offset gathers and the flatness of angle gathers, the results at iteration 9 are satisfactory (Figure 7e-7h). We observe that the strong amplitudes on the far offset for shallower events (Figure 7e and 7f) are unaffected by the horizontal warping. The reasons for this may come from many aspects. First of all, the gradient subject to the horizontal warping on these image artifacts is not constructively interfered. On the other hand, the inversion scheme we impose is not trying to remove these artifacts as opposed to the schemes, where there is a global objective function to which reducing such artifacts results a)

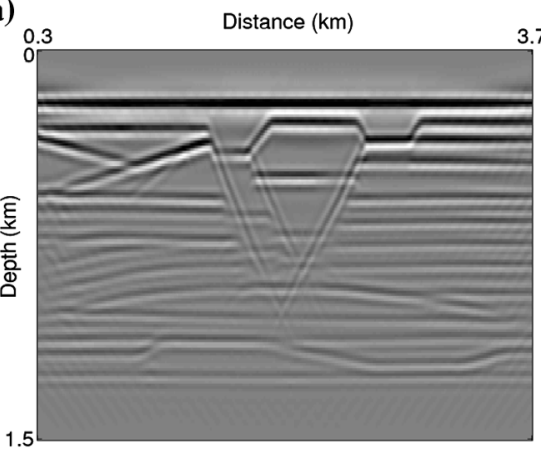

b)

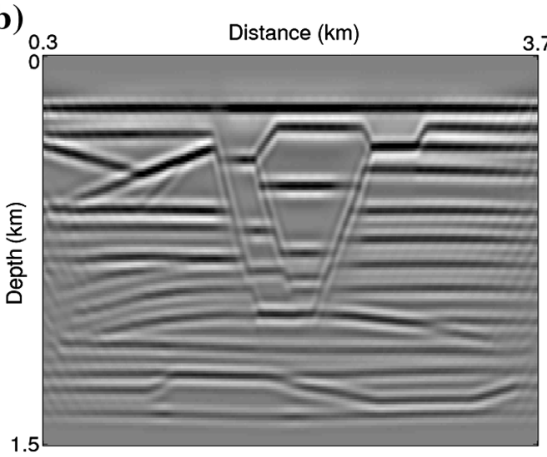

c)

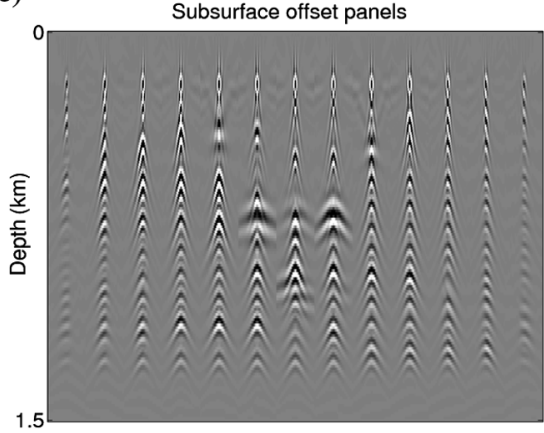

d)

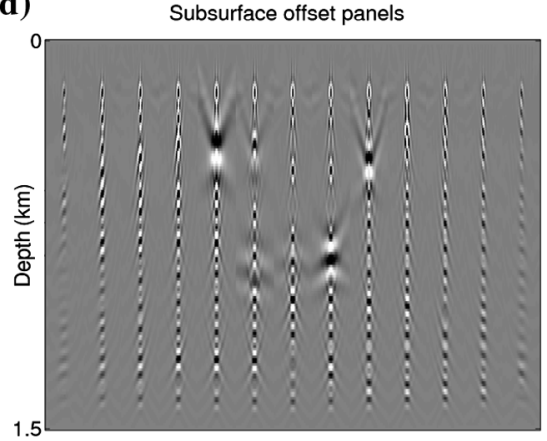

e)

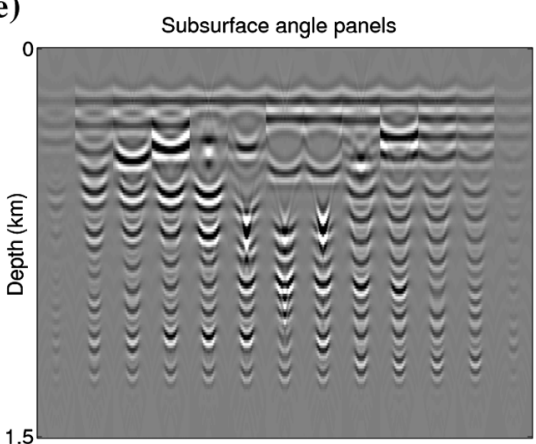

f)

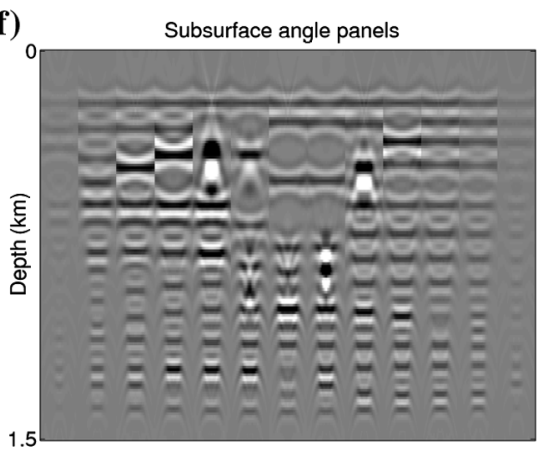

Figure 5. (a) Initial image, (b) image at iteration 20, (c) initial subsurface offset gathers, (d) subsurface offset gathers obtained at iteration 20, (e) initial angle gathers, and (f) angle gathers obtained at iteration 20. All gathers are collocated and evenly distributed horizontally across the model. 
in decreasing the objective function value. This phenomenon is very different from a conventional DS-type inversion, in which the faroffset amplitudes are reduced at the cost of changing the velocity (Shen, 2013).

\section{Example 4: Salt body inversion}

The real challenge of velocity model building in the Gulf of Mexico is the inversion of the salt body itself. Subject to automatic wave-equation schemes, this example is one of the attempts to invert for the salt body from an initial model that is devoid of salt. The true model is shown in Figure 8a, which has the same dimensions as example 3. The difference is that there we introduce a salt in the middle to replace the one in the bottom-right corner of example 3 . The salt velocity is $4800 \mathrm{~m} / \mathrm{s}$, and the surrounding velocity is $1500 \mathrm{~m} / \mathrm{s}$ for water increased to $3300 \mathrm{~m} / \mathrm{s}$ to the bottom of the model. The data illustrated in Figure $8 \mathrm{~b}$ are full-waveform simulated with a sampling rate of $16 \mathrm{~ms}$ for $8 \mathrm{~s}$, which reaches a cut-off frequency of $31.25 \mathrm{~Hz}$. The acquisition geometry is the same as example 3. Again, the direct arrivals and a major part of the diving waves are strongly reduced with an application of the obliquity factor to the data.

The left-side salt in the true velocity does not generate any base salt reflection or internal reflection that arrives at the surface. From an imaging perspective, the same image can be obtained with an effective true velocity, in which the left-side salt is replaced by the sediment velocity. This is shown in Figure $8 \mathrm{c}$ for the effective true velocity and Figure $8 \mathrm{~d}$ for the corresponding image. For realistic considerations, we make the initial model largely homogeneous laterally and devoid of salt (Figure 8e). This poses two challenges: First, the missing right-side salt introduces velocity errors much beyond a Born approximation in the sense that the difference between the current image and the true image is not linearly related to the difference between the current velocity and the true velocity, subject to the full bandwidth that the data provide, and second, the sedimentary velocity is incorrect. The effect of these is immediately seen in the initial image shown in Figure 8f. The objective is to bring the salt back and produce the correct sedimentary velocity above the salt by automatic velocity updating with horizontal warping.

The gradient and the Hessian (diagonal approximation) of the first iteration are shown in Figure $9 \mathrm{a}$ and $9 \mathrm{~b}$, respectively. We make the following observations:

1) The fluctuations of the gradient and the Hessian above the top salt, although not oscillating from positive to negative values, show clear wavepath imprints induced by the tip of the salt.

2) These wavepaths imprints presented in the gradient and the Hessian share similar patterns. However, a simple division of the gradient by the Hessian could not remove them.
3) The gradient in the region corresponds to the missing salt has homogeneous values, but it is small in amplitude. On the other hand, the Hessian in the same area also has small but homogeneous values. The ratio between the two effectively boosts the values in areas in which the most significant physical velocity update is expected.

4) Below the salt, due to little illumination, the amplitude of the Hessian is exceedingly small.

These observations are not present or are not as evident as in example 3. Item 3 works in favor for the inversion. An inverse Hessian correction, as described in equation 16 or equation 21 would adjust the gradient amplitude efficiently in one iteration where the salt is missing, which would normally cost many quasi-Newton iterations. The issues presented in items 1 and 2 are treated by a sequence of smoothing operators jointly applied with image-domain regularization $K$ (Appendix B). The characteristic difference in this example is that the smoothing operator is not simplified as the identity, as in example 3. a)

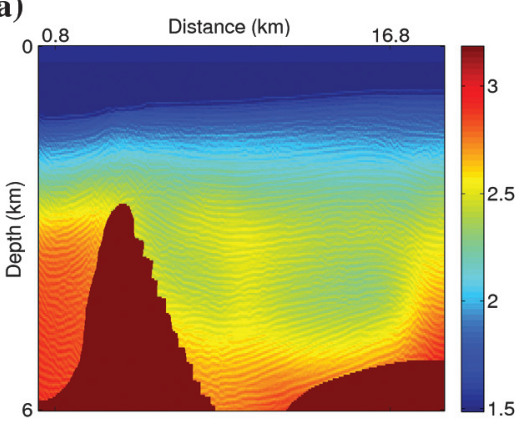

b)

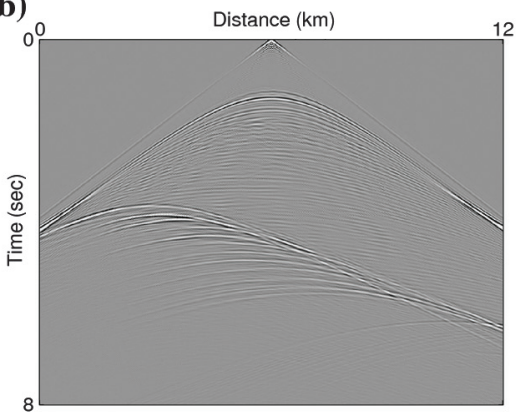

c)

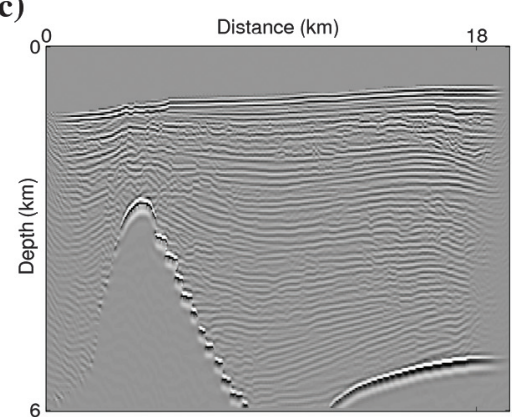

d)
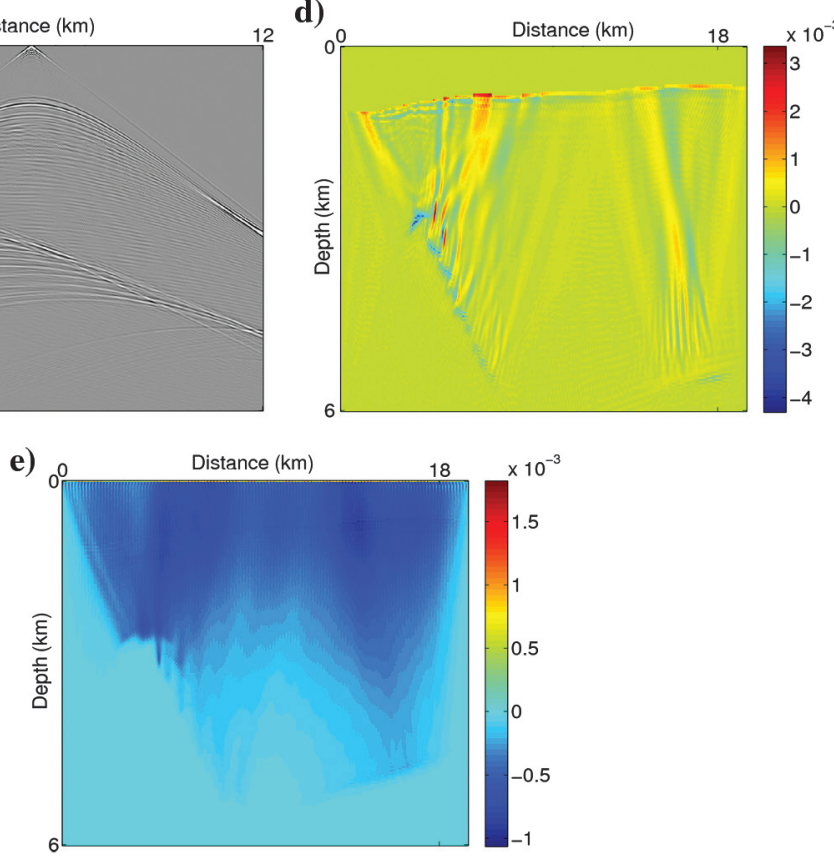

Figure 6. (a) True velocity that contains salt bodies, the sedimentary velocity, and reflectors as velocity perturbations. (b) Split-spread shot data. (c) Image obtained with true sedimentary velocity and salt bodies. (d) Conventional DS gradient, obtained at the initial velocity shown in Figure 7a, oscillates above the top salt. The section above the ocean bottom is muted. (e) The gradient of horizontal warping, obtained at the initial velocity shown in Figure 7a, does not oscillate above the top salt. 
A careful thresholding on the diagonal of Hessian as the denominators to avoid dividing by exceedingly small numbers is required to address item 4. We list all treatments applied: a protected division to the ratio between the gradient and the Hessian, followed by negating the sign, replacing zeros for the water column, and scaling by a constant; the final result that effectively represents $d c / d t$ is shown in Figure 9c. The most significant updates are in the missing salt area and the area above the tip of the left-side salt. The deeper part of the model shows a clear cut-off value. This is due to the protected division of Hessian. In the second iteration, the gradient and the approximated diagonal of the Hessian vary fairly significantly, but the ratio between them, with the treatments described above, remains concentrated in the area of the missing salt (Figure 9d). At the same time, the sedimentary update above the tip of the salt is noticeably reduced. These results suggest that the Hessian-projected gradient is capable of producing strong and coherent local velocity updates iteratively in a region that is far beyond the Born approximation. This is not surprising because the diagonal Hessian correction to the gradient is essentially an economic approximation to Newton's step.

For a conventional DS-type inversion, one needs to capture the entire moveout of the image in offset. This necessarily includes the range over which the image decays to zero and causes a huge storage and computational burden to the inversion because the cost of the crosscorrelations and the Born scatterings in offset increases linearly on the number of offsets. For horizontal warping, due to the sharp truncation in offset, we actually make use of an offset range much less than what is required to capture the entire moveout. This can be seen from the far offset at the base-of-salt images, where the energy does not nearly decay relative to that of the zero offset. We comment on this phenomenon as follows: The unregularized conventional DS methods (Shen and Symes, 2008) create a dissipative system in which the energy of image is diffused across all offsets. This point is analyzed in the "Discussion" section. In case of a small offset range, the conventional DS objective function value may decrease due to energy diffusions into unrecorded large offsets. When a)

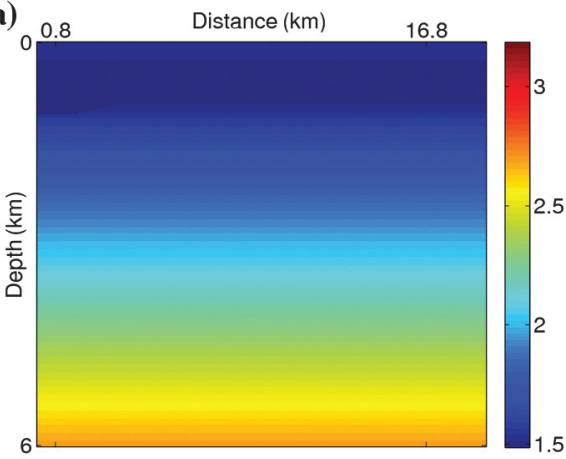

b)

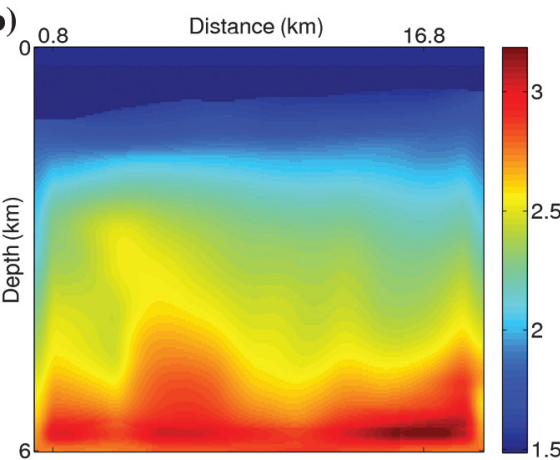

c)

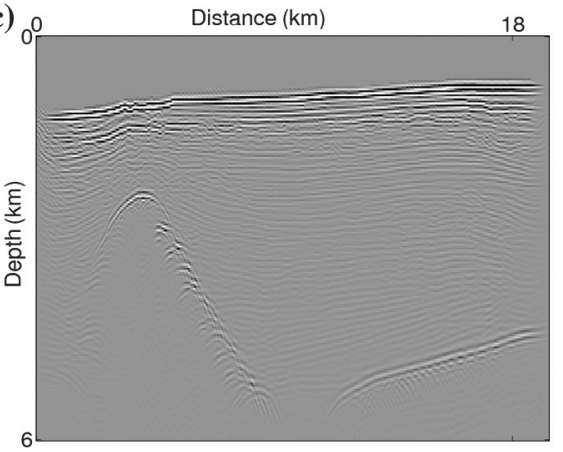

d)

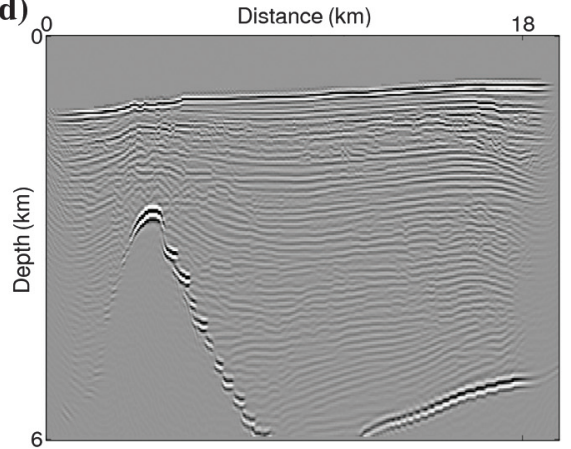

e)

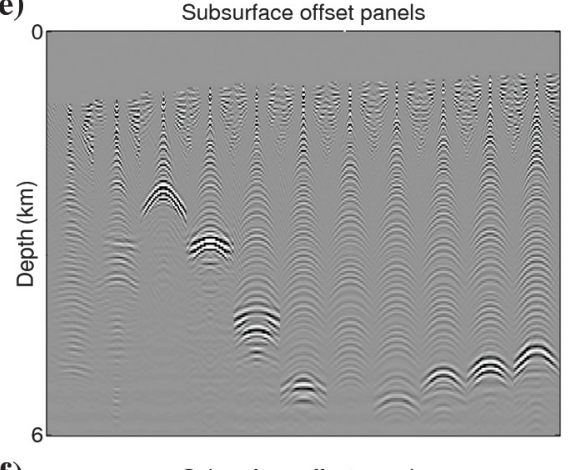

f)

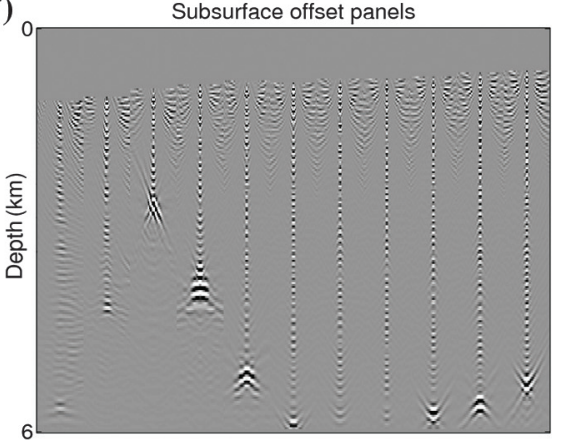

g)

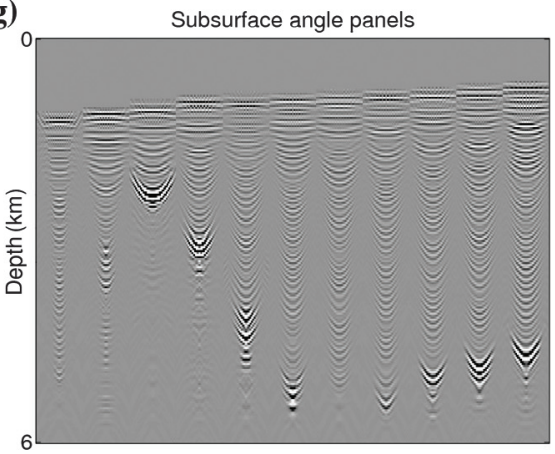

h)

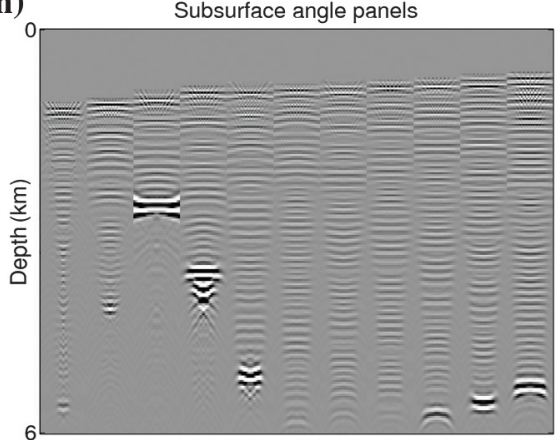

Figure 7. (a) Initial velocity, (b) velocity at iteration 9, (c) initial image, (d) image at iteration 9, (e) initial offset gathers, (f) offset gathers at iteration 9, (g) initial angle gather, and (h) angle gathers at iteration 9. All gathers are collocated at positions with an even distribution horizontally. 
the offset range is sufficiently large, the penalty function starts to prevent energy from diffusing into large offsets: The process of collapsing to zero offset overrules the process of dissipating the energy across the offsets. This is one explanation of why conventional DS needs large offset ranges. The diffusion process does not exist in an ideal offset-domain warping (such as total warping described in the "Limitations of horizontal contraction" section), in which the image registered at every offset produces a uniform phase shift toward the focus. The aforementioned energy argument does not directly influence the focusing flow in the image. Horizontal warping, as an approximation to total warping, inherits this feature with the exception of the lensing velocity structure.

Another differentiating technique, that we improve in comparison with example 3 , is the inclusion of the stack power to image residual:

$$
-\partial_{|h|} I \Delta h-\alpha S(h) I
$$

where $\alpha$ is a positive number that is dynamically adjusted between iterations and $S(h)$ is a semipositive definite diagonal operator in $h$ that maximizes its singular value at $h=0$. We shall call $S$ the stack-power operator. The skew-symmetric operator $\partial_{|h|}$ preserves the energy in a dynamic system. This is very different from the conventional DS operators, such as $h^{2}$ or $-\partial^{2} / \partial h^{2}$ as

b) discussed in Table 1, in that the latter lose energies. A conventional solution to preserve the energy in a DS-type inversion is to include a stack-power term, so that velocities that reduce the energies of the
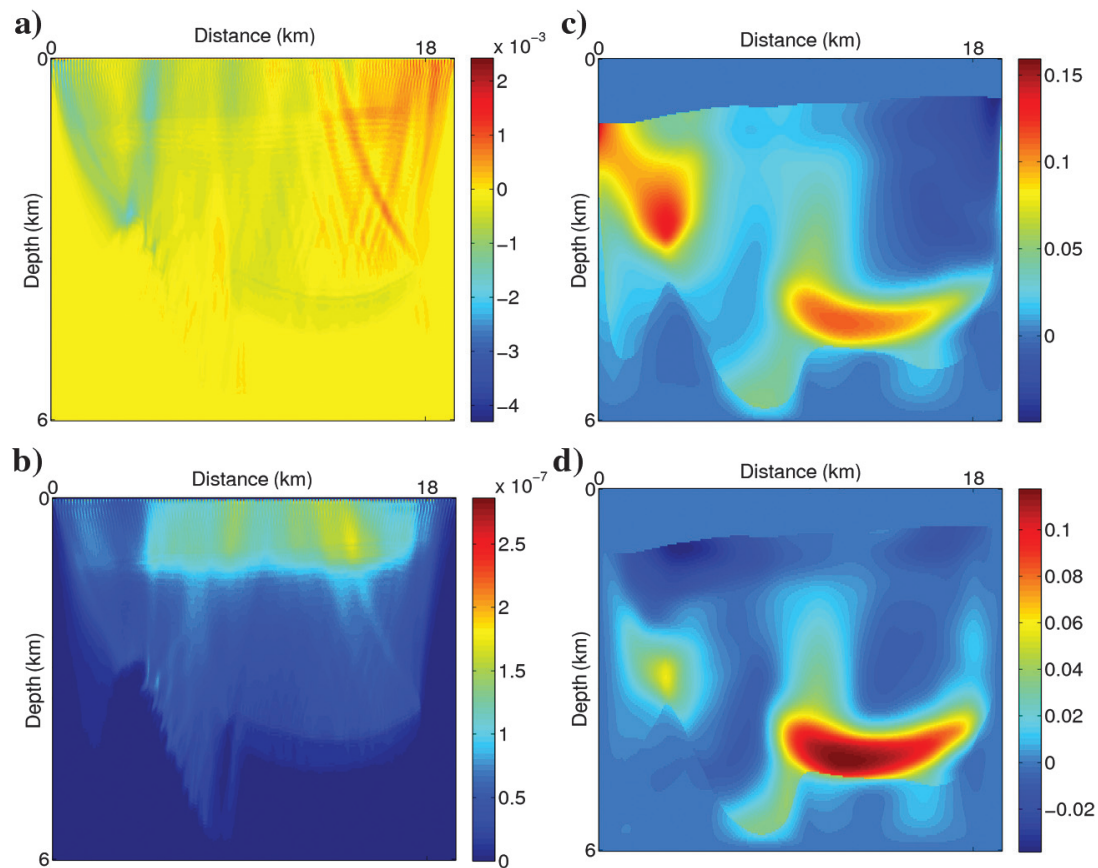

Figure 9. (a) Gradient at the first iteration, (b) approximated diagonal of Hessian at the first iteration, (c) Hessian corrected gradient at the first iteration, and (d) Hessian corrected gradient at the second iteration. a)

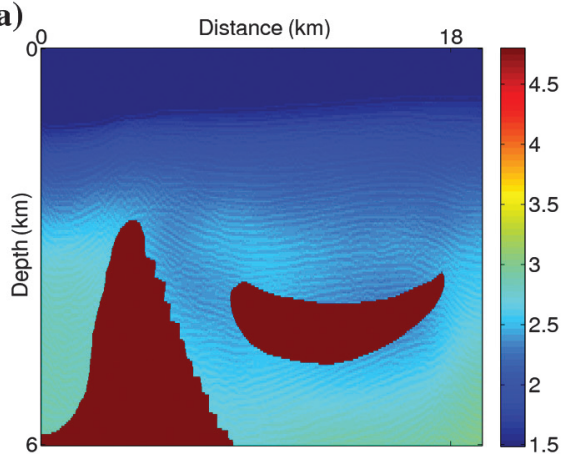

b)

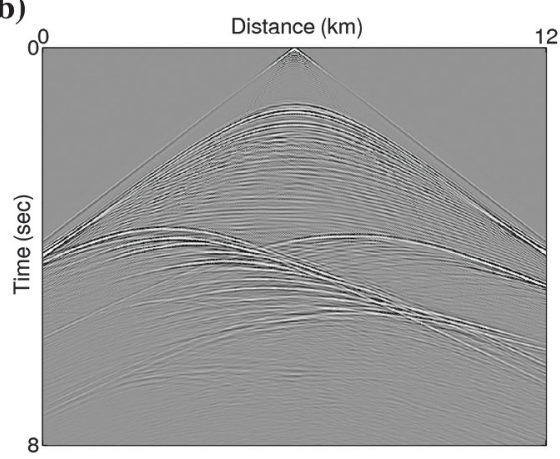

c)

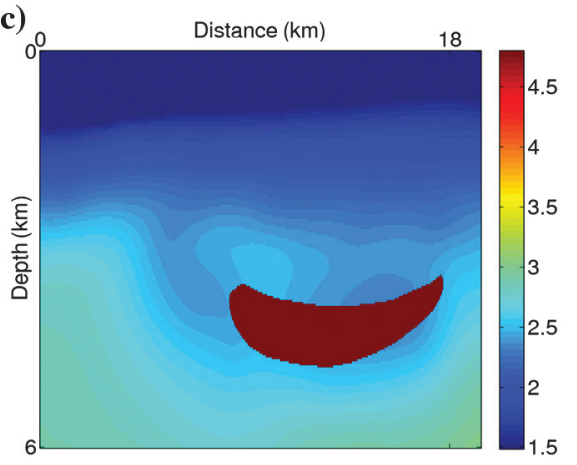

d)

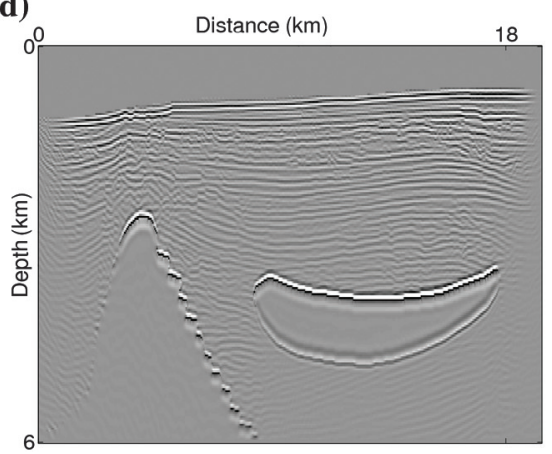

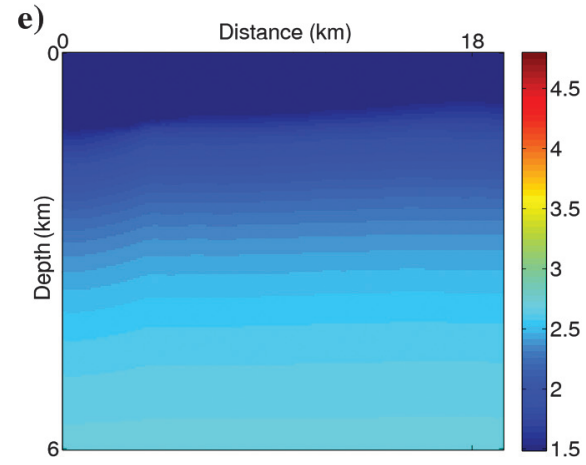

f)

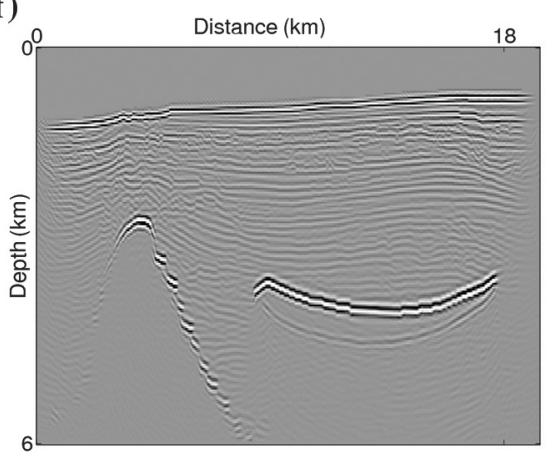

Figure 8. (a) Simulation velocity. The salt body velocity is $4800 \mathrm{~m} / \mathrm{s}$. The surrounding sediment velocity is approximately $2500 \mathrm{~m} / \mathrm{s}$. (b) Fullwaveform simulation with the direct arrival tapered by an application of the obliquity factor. (c) Effective true velocity. (d) Image obtained from the effective true velocity. (e) Initial velocity that is devoid of salt and incorrect in the sedimentary section. (f) Initial image. 
image in offset are rejected (Shen and Symes, 2008; Shen, 2012). It is also noted that the stack-power term can provide velocity information if the current velocity is sufficiently close to the true. In general, including a stack-power term in the image residual always helps to stabilize the inversion. We further have the advantages that the algorithm does not require $\alpha$ to be fixed nor to be differentiable to velocity. The $\alpha$ value is dynamically adjusted, so that the $l_{2}$-norm of $\alpha S I$ is $15 \%$ of the $l_{2}$-norm of $\partial_{|h|} I \Delta h$ for each iteration.

Twenty iterations of inversion result in a velocity (Figure 10a) fairly similar to the effective true velocity. The inverted salt not only has a well-defined boundary, but the center of which reaches a

Table 1. Column 2, differential semblance in angle or surface offset, where $h$ denotes the specular angle or surface offset; column 3, differential semblance in subsurface offset, where $h$ is the offset and $p^{2}(h)$ penalizes the image supported away from $h=0$ and annihilates the image at the correct velocity; and column 4 , an example of horizontal contraction in the subsurface offset, where the compression is exponentially scaled.

Surface-offset DS Subsurface-offset DS Subsurface horizontal contraction

\begin{tabular}{lccc}
\hline Image residual & $-\partial_{h}^{2} I$ & $p^{2}(h) I$ & $-|h| \partial_{|h|} I$ \\
Image evolution & $\frac{\partial I}{\partial t}=\partial_{h}^{2} I$ & $\frac{\partial I}{\partial t}=-p^{2}(h) I$ & $\frac{\partial I}{\partial t}=|h| \partial_{|h|} I$ \\
Solution & Constant as $t \rightarrow \infty$ & $I=e^{-p^{2}(h) t} I_{0}$ & $I=I_{0}\left(e^{t} h\right)$ \\
\hline
\end{tabular}

a)

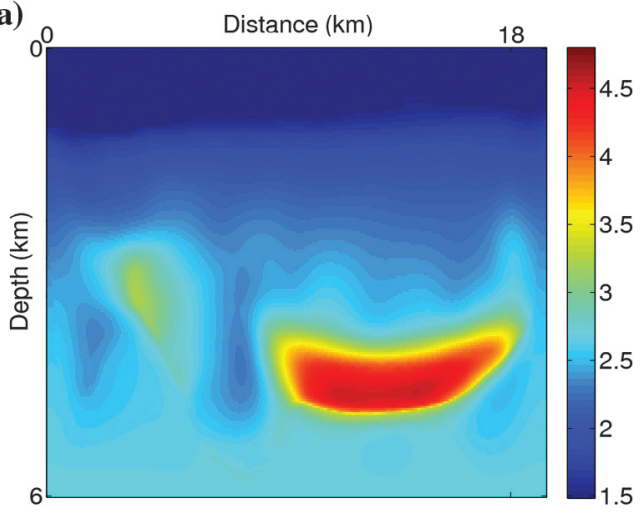

b)

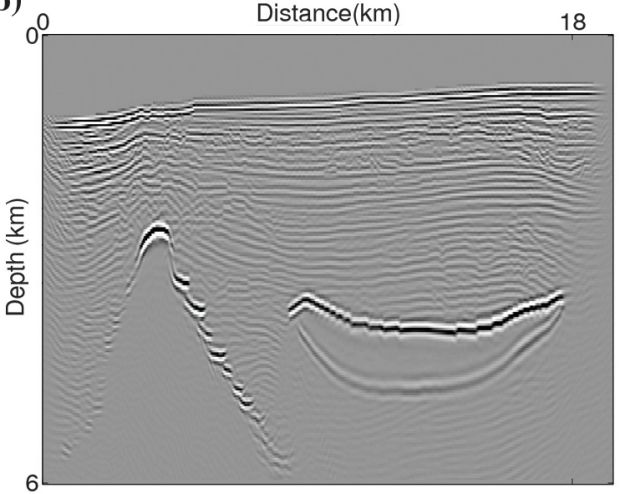

Figure 10. (a) Velocity obtained at iteration 20 and (b) image obtained at iteration 20 using the velocity shown in panel (a). velocity higher than $4500 \mathrm{~m} / \mathrm{s}$, increased almost $100 \%$ from its initial value. The corresponding image (Figure 10b) also resembles the correct image obtained from the true velocity. Once more, we show the gathers of the initial and the final iterations as the velocity quality indicator. All gathers are collocated at the same positions that distribute over the horizontal span. The image in offset for the base of salt, illustrated in Figure 11a surrounded by the orange ellipse, is well spread laterally due to the missing of the salt body. The top-salt event circled on the left is also not focused due the sediment velocity error. The resulting gathers in offset (Figure 11b) and angle (Figure 11d) at iteration 20 show significant improvement, not only for the base of salt but also for some sedimentary events.

A comparison of nominal results obtained with and without stack-power terms is shown in Figure 12. It is observed that the velocity with the stack-power term used is slightly better than without. Consequently, the image gather in the angle with the stack-power term is also visibly flatter in the two panels on the left (Figure 12c) than the right (Figure 12d). With the exception of Figure 12, all results shown in this section are obtained with stack power.

\section{DISCUSSION}

An image residual is really a direction vector in image space, i.e., for each image, an increment moving it closer to focus. Such assignment of a direction to each point in a space amounts to a differential equation in the space, which in turn gives rise to an evolution flow. Table 1 describes the image evolution flow induced by several image residual constructions. The first two columns describe gradient flows induced by two versions of differential semblance, as described by Symes (2008). That is, the differential semblance image residual is by itself the gradient of an objective function. The last column describes the horizontal contraction residual; it is not the gradient of an objective function.

We use $h$ in column 2 of Table 1 to indicate the surface offset or subsurface angle. The DS algorithm in the angle or surface offset can be posed to minimize $J=\frac{1}{2}\left\|\partial_{h} I\right\|^{2}$. A perturbation of $J$ leads to the image residual $R=\partial_{h}^{*} \partial_{h} I=-\partial_{h}^{2} I$. Considering an evolution flow in $t$ induced by the image residual, we can write $\frac{\partial I}{\partial t}=\partial_{h}^{2} I$. Here, the additional minus sign on the residual is to force the image to evolve toward the direction that decreases the objective function. The equation is recognized as a heat equation. The solution at $t \rightarrow$ $\infty$ approaches to constant in unbounded domain. In a similar way, we can set up the image residual and the evolution equation for the subsurface DS algorithm. As shown in column 3, $p(h)$ is a polynomial penalizing factor in offset. The solution of evolution equation can be expressed using the initial image $I_{0}$ as $e^{-p^{2}(h) t} I_{0}$. Although it is focused at $h=0$ when $t \rightarrow \infty$, no information for $h \neq 0$ in $I_{0}$ is present in the solution. In the last column, the image residual is defined as the current image less the image that is slightly improved or more focused. It is consistent with the convention used before: An image residual is to increase the objective function if by minimizing the objective function a better image is found. Here, we see that the solution at $t \rightarrow \infty$ is indeed concentrated at $h=0$ with all information in offset presented in $I_{0}$. 
a)

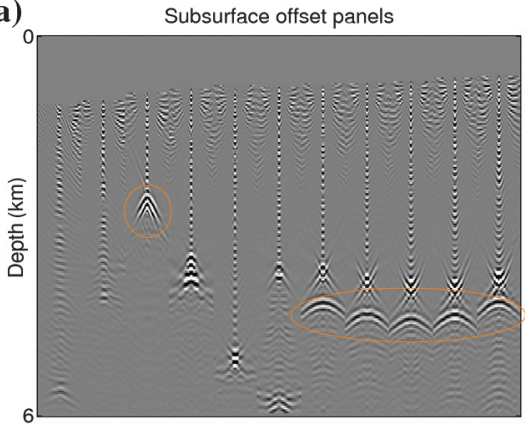

b)

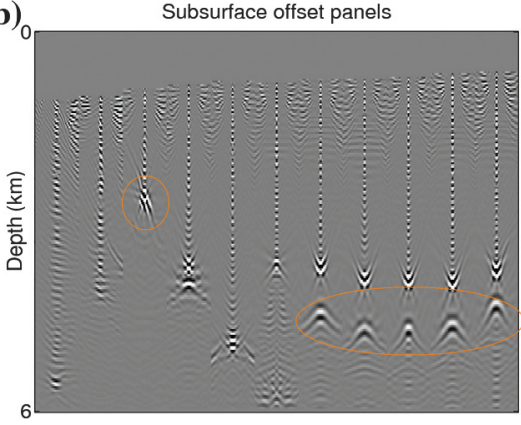

c)

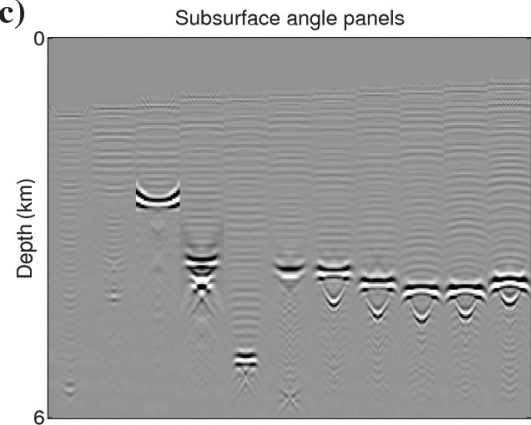

d)

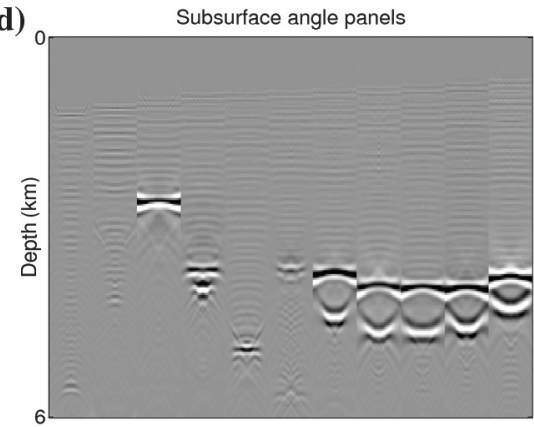

Figure 11. Comparison of image gathers before and after the inversion: (a) Initial subsurface offset gathers, (b) subsurface offset gathers at iteration 20, (c) initial angle gathers, and (d) angle gathers at iteration 20. All gathers are collocated at positions evenly distributed horizontally. The circled areas in panels ( $a$ and $b$ ) show the most evident differences in offset for before and after inversion, respectively. The images in the angle are correspondingly improved in the same areas. a)

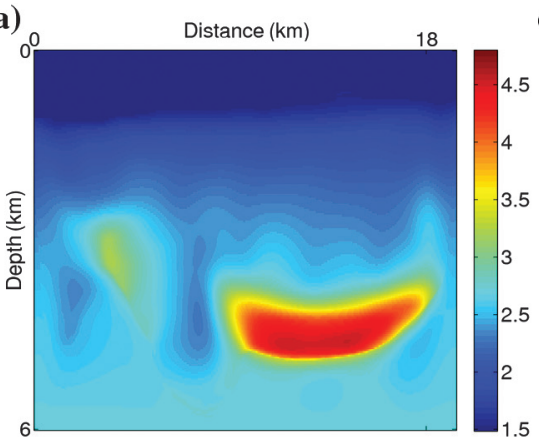

b) 0

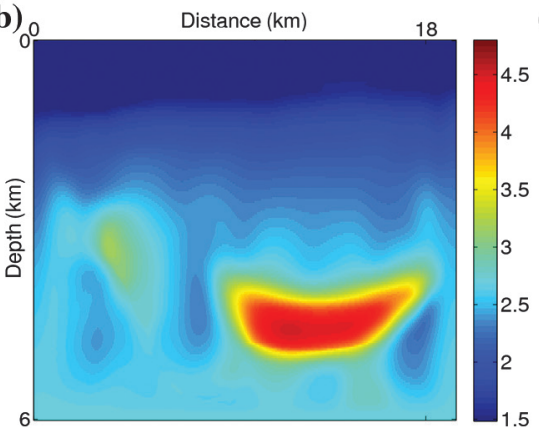

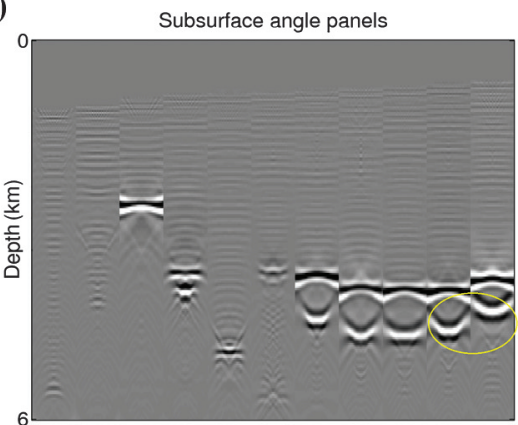

d)

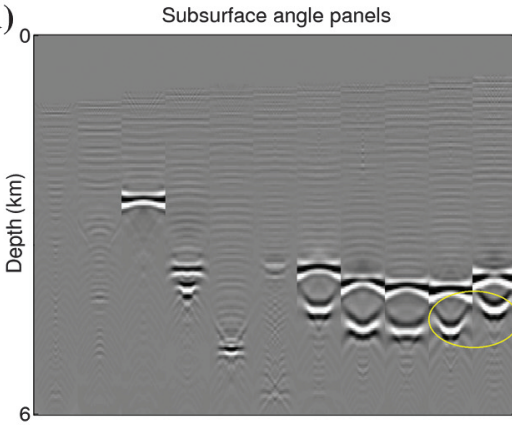

Figure 12. Comparison of inversion results obtained with and without stack power in the inversion: (a) velocity output at iteration 20 with stack power, (b) velocity output at iteration 20 without stack power, (c) angle gathers at iteration 20 with stack power (Figure $11 \mathrm{~d}$ repeated), and (d) angle gathers at iteration 20 without stack power. The bottomright angle gathers surrounded by the yellow circle of panel (d) are slightly worse than panel (c).
All three flows have the same asymptotic limit sets, that is, focused images, and that is why all three generate constructive velocity updates via least-squares projection (equation 5), in some circumstances. The surface offset (or other acquisition parameter) differential semblance image residual results in the heat equation in offset, whose trajectories limit to images constant in offset, which is the focusing criterion for this type of image space. The related velocity update fails in the presence of multipathing, whereas the velocity updates generated by the differential semblance image residual in horizontal subsurface offset (column 2) remain constructive in strongly refractive velocity models, so long as the relevant rays do not turn horizontal (Symes, 2008). The flows for the differential semblance and the $|h|$ scaled horizontal contraction (column 3) image residuals are limited to images focused at zero offset, but in different ways: The differential semblance flow (column 2) "forgets" the initial image energy at a nonzero offset, whereas the horizontal contraction flow incorporates it into the zero-offset limit. This difference may be related to the presence, versus the absence, of artifacts described earlier in the velocity updates derived from differential semblance, respectively, horizontal contraction, for subhorizontal broken reflectors. On the other hand, differential semblance has performed well for velocity lens examples (Shen and Symes, 2008), whereas our examples on the lens reveal that horizontal contraction apparently leads to velocity updates of the wrong sign. The fundamental solution in warping to avoid generating the wrong sign for velocity updates with a refracting structure requires total warping as described in the "Limitations of horizontal contraction" section where a vertical shift must be incorporated. This means that the infinitesimal generator is no longer purely horizontal, which is a subject beyond the scope of this paper.

\section{CONCLUSION}

We have shown that several recently suggested velocity update formulas, including variants of differential semblance, may be cast in the form of the least-squares projection into velocity space from an image residual. We also show how to implement the horizontal contraction velocity update efficiently beyond the function-gradient quasi-Newton's scheme and given a sequence of synthetic examples leading ultimately to salt body inversion. Our work gives a dynamic systems interpretation to the image based velocity inversion. An infinitesimal motion in the image gives rise to an infinitesimal motion in velocity through the tangent map from the image to velocity treated as the generalized inverse. We verify that horizon- 
tal contraction image residuals lead to smoother velocity updates and faster convergence, in comparison with classical differential semblance. Although the prescribed horizontal contractive image increment imposes limitations in the presence of refracting velocity structures, many image residuals besides differential semblance and horizontal contraction define flows that limit to focused images, hence potentially generating useful velocity updates. Most of these possibilities remain to be explored.

\section{ACKNOWLEDGMENTS}

Without H. Kuehl's encouragement and promotion of discussions, this work could not have reached its current stage. The first author wants to thank his former colleague R. Cook and S. Jin for many insightful discussions. R.-E. Plessix carefully reviewed the work and provided many detailed recommendations, which the first author sincerely appreciates. Q. Cao helped to review the draft. F. ten Kroode supported this work. We want to thank three anonymous reviewers and S. Operto for proofreading and many valuable suggestions.

\section{APPENDIX A}

\section{DIFFERENTIAL OF IMAGE WITH RESPECT TO VELOCITY AND ITS ADJOINT IN WAVE-EQUATION FORMULATION}

In this appendix, we will formulate the Fréchet derivative of image with respect to velocity and its adjoint. The main difficulty is the adjoint formulation. We start by hiding the details of how the image is formed (typically through correlation of source and receiver wavefields in the subsurface offset), and we write the image in subsurface offset as

$$
I=I(p, q)
$$

Here, $I$ is the image in the subsurface offset (to be called "image" for brevity), $p$ is the forward source field that satisfies $L p=f$ where $L$ is the wave operator with proper boundary and initial conditions, $f$ is the source wavelet, and $q$ is the backward receiver field that satisfies $L^{*} q=d$ with $d$ being the reflection data, and the superscript $*$ indicates the transpose in time. The Fréchet derivative of the image with respect to velocity $c$ can be written by the chain rule as

$$
\frac{\partial I}{\partial c}=\frac{\partial I}{\partial p} \frac{\partial p}{\partial c}+\frac{\partial I}{\partial q} \frac{\partial q}{\partial c} .
$$

Let $L^{-1}$ and $L^{-*}$ be, respectively, the inverse of $L$ and $L^{*}$, we have the following relations:

$$
\begin{aligned}
p & =L^{-1} f, \quad q=L^{-*} d \\
\frac{\partial L^{-1}}{\partial c} & =-L^{-1} \frac{\partial L}{\partial c} L^{-1}, \quad \frac{\partial L^{-*}}{\partial c}=-L^{-*} \frac{\partial L^{*}}{\partial c} L^{-*} .
\end{aligned}
$$

Taking the adjoint of equation A-2 using the above relations, we obtain

$$
\begin{aligned}
\left(\frac{\partial I}{\partial c}\right)^{*}= & -\left(L^{-1} f\right)^{*} \frac{\partial L^{*}}{\partial c} L^{-*}\left(\frac{\partial I}{\partial p}\right)^{*} \\
& -\left(L^{-*} d\right)^{*} \frac{\partial L}{\partial c} L^{-1}\left(\frac{\partial I}{\partial q}\right)^{*} .
\end{aligned}
$$

The first term on the right side of equation A-4 can be interpreted as follows: The $\left(L^{-1} f\right)^{*}$ is the forward source wavefield transposed in time; the $(\partial I / \partial p)^{*}$ is a transformation that maps a quantity in the image domain to another quantity in the image domain; the concatenation of $L^{-*}$ and $(\partial I / \partial p)^{*}$ forms $L^{-*}(\partial I / \partial p)^{*}$, which serves as an adjoint state wavefield solver that is to be applied to an image-domain Born source - the result of this application is a backward Born receiver wavefield; and $(\partial L / \partial c)^{*}$ provides the weight (metric) for the correlation (inner product) between the forward source field and the backward Born receiver field. Likewise, the second term on the right side of equation A-4 is to be interpreted as the correlation between a forward wavefield and the corresponding adjoint-state Born wavefield, but the roles of the source and receiver are switched relative to the first term. For a second-order-in-time scalar wave equation propagator, $\partial L / \partial c$ has a simple expression:

$$
\frac{\partial L}{\partial c}=-\frac{2}{c^{3}} \partial_{t}^{2}
$$

The operator $(\partial I / \partial c)^{*}$ acting on some image-domain quantity, say, an image residual, is the subject matter of the discussion. Let $u$ be some image-domain quantity that is supported in $h$ the (inline horizontal) subsurface offset and $x$ the common image point, we write

$$
u=u(x, h ; p, q)
$$

We are interested in the formulation of $(\partial I / \partial c)^{*}$ acting on $u$, namely, $(\partial I / \partial c)^{*} u$. Substituting equation A-4 to the expression $(\partial I / \partial c)^{*} u$, we can see the need to interpret the meanings of $(\partial I / \partial p)^{*} u$ and $(\partial I / \partial q)^{*} u$. It is now necessary to raise the details of how the image is formed. One of many versions of imaging condition is

$$
I(x, h ; p, q)=\int \partial_{t}^{2} p(x-h, t) q(x+h, t) \mathrm{d} t,
$$

where $t$ is the forward propagation time. Subject to the above imaging condition, we derive

$$
\begin{aligned}
& \left(\left(\frac{\partial I}{\partial p}\right)^{*} u\right)(x, t)=\left(\partial_{t}^{2}\right)^{*} \int d h q(x+2 h, t) u(x+h, h), \\
& \left(\left(\frac{\partial I}{\partial q}\right)^{*} u\right)(x, t)=\left(\partial_{t}^{2}\right)^{*} \int d h p(x-2 h, t) u(x-h, h) .
\end{aligned}
$$

The operator $\partial_{t}^{2}$ is self-adjoint for band-limited signals. Combining the expression $(\partial I / \partial c)^{*} u$, equations $\mathrm{A}-8$ and $\mathrm{A}-4$, we arrive at

$$
\begin{aligned}
\left(\frac{\partial I}{\partial c}\right)^{*} u= & -p^{*} \frac{\partial L^{*}}{\partial c} L^{-*} \int \partial_{t}^{2}\left(T_{2 h} q\right)\left(T_{h} u\right) \mathrm{d} h \\
& -q^{*} \frac{\partial L}{\partial c} L^{-1} \int \partial_{t}^{2}\left(T_{-2 h} q\right)\left(T_{-h} u\right) \mathrm{d} h .
\end{aligned}
$$


Here, $T_{h}$ is a spatial translation operation in $x, T_{h} p(x, \ldots)=$ $p(x+h, \ldots)$. Equation A-9 can also be viewed as a matrix-vector multiplication, where time is row indexed and the "**" sign indicates transposition in time. This completes the formulation to adjoint of the Fréchet derivative, without specifying the details of $L$. In other words, it covers the RTM formulation in the subject matter. The forward Fréchet derivative is straight forward. Under the same imaging condition A-7, we write $(\partial I / \partial c)$ acting on the velocity perturbation $\delta c$ as

$$
\begin{aligned}
\left(\frac{\partial I}{\partial c}\right) \delta c= & \partial_{t}^{2} \int \mathrm{d} h \delta p(x-h, t ; \delta c) q(x+h, t) \\
& +\partial_{t}^{2} \int \mathrm{d} h p(x-h, t) \delta q(x+h, t ; \delta c),
\end{aligned}
$$

where the Born source field is expressed as

$$
\delta p(\ldots ; \delta c)=-L^{-1}\left(\frac{\partial L}{\partial c} \delta c\right) L^{-1} f
$$

and the Born receiver field is expressed as

$$
\delta q(\ldots ; \delta c)=-L^{-*}\left(\frac{\partial L^{*}}{\partial c} \delta c\right) L^{-*} d
$$

Equations A-9 and A-10 constitute the main computational operations required for the velocity inversion method described in the main text. Especially, the concatenation of $(\partial I / \partial c)^{*}$ and $(\partial I / \partial c)$ acting on a constant velocity field subject to the conditioning operator $K$ :

$$
\tilde{H}_{d}=\left(\frac{\partial I}{\partial c}\right)^{*} K\left(\frac{\partial I}{\partial c}\right) 1 c
$$

becomes the approximated diagonal of the Hessian.

\section{APPENDIX B}

\section{REGULARIZATION BY CHAIN RULE}

A vector in the image connects to a vector in the velocity through the chain rule:

$$
\frac{d c}{d t}=\frac{\partial c}{\partial I} \frac{d I}{d t}=\left(\frac{\partial I}{\partial c}\right)^{-1} \frac{\partial I}{\partial t}
$$

Replacing $(\partial I / \partial c)^{-1}$ by a (model space) generalized inverse

$$
\left(\frac{\partial I}{\partial c}\right)^{-1}=\left[\left(\frac{\partial I}{\partial c}\right)^{*} \frac{\partial I}{\partial c}\right]^{-1}\left(\frac{\partial I}{\partial c}\right)^{*}
$$

we arrive at the main content of this paper:

$$
\frac{d c}{d t}=\left[\left(\frac{\partial I}{\partial c}\right)^{*}\left(\frac{\partial I}{\partial c}\right)\right]^{-1}\left(\frac{\partial I}{\partial c}\right)^{*} \frac{d I}{d t},
$$

which is as follows: A rule to evolve the image gives rise to a rule to evolve velocity. Now, we impose certain constraints to image via $I_{l}=L I$ and velocity via $c_{m}=M c$, where $L$ and $M$ are linear operators through which the constraints on the image and velocity, respectively, are imposed. Now, suppose we are interested in a velocity that is subject to these constraints. We derive

$$
\begin{aligned}
\frac{d c}{d t} & =\frac{\partial c}{\partial c_{m}} \frac{\partial c_{m}}{\partial I_{l}} \frac{\partial I_{l}}{\partial I} \frac{d I}{d t}, \\
& =\left(\frac{\partial I_{l}}{\partial c_{m}} \frac{\partial c_{m}}{\partial c}\right)^{-1} \frac{\partial I_{l}}{\partial I} \frac{d I}{d t}, \\
& =\left(\frac{\partial I_{l}}{\partial I} \frac{\partial I}{\partial c_{m}} \frac{\partial c_{m}}{\partial c}\right)^{-1} \frac{\partial I_{l}}{\partial I} \frac{d I}{d t}, \\
& =\left(L \frac{\partial I}{\partial c_{m}} M\right)^{-1} L \frac{d I}{d t}, \\
& =\left(M^{*}\left(\frac{\partial I}{\partial c_{m}}\right)^{*} L^{*} L\left(\frac{\partial I}{\partial c_{m}}\right) M\right)^{-1} M^{*}\left(\frac{\partial I}{\partial c_{m}}\right)^{*} L^{*} L \frac{d I}{d t}
\end{aligned}
$$

The first $=$ sign is the chain rule expansion, the second $=$ sign in dicates to which subproblem the least-squares are imposed, the fourth $=$ sign inserts the constraints in image and velocity, and the last $=$ sign substitutes the generalized inverse for the inverse operator. The foregoing equation reduces to equation B-1 if $M$ and $L$ are the identity, and it reduces to equation (21) if $M$ is the identity, recalling $K=L^{*} L$. For practical purposes, $K$ is regarded as an amplitude balancing operator. We further require that $M$ be self-adjoint $M=M^{*}$. The intended action by $M$ is smoothing, so that $c_{m}$ is smoother than $c$. If we are interested in updating $c_{m}$, the formula becomes

$$
\frac{d c_{m}}{d t}=M\left(M\left(\frac{\partial I}{\partial c_{m}}\right)^{*} K\left(\frac{\partial I}{\partial c_{m}}\right) M\right)^{-1} M\left(\frac{\partial I}{\partial c_{m}}\right)^{*} K \frac{d I}{d t}
$$

The implementation of equation B-5 is interpreted as follows: For horizontal contraction, the rate of change of the image is governed by $d I / d t=|h|^{n} \partial_{|h|} I$. The left side is treated as the image residual, and the image-domain regularization $K$ is applied to balance the amplitude. The result is then passed to the gradient calculation followed by the smoothing operation $M$. We shall call this intermediate result the $M$ smoothed gradient subject to $K$. The diagonal approximation as described in the "Method" section is triggered by a velocity perturbation $1_{c}$, and $M 1_{c}=1_{c}$. So, the diagonal Hessian approximation becomes $\left(\partial I / \partial c_{m}\right)^{*} K\left(\partial I / \partial c_{m}\right) 1_{c}$ followed by the smoothing by $M$. We shall call this second intermediate result the $M$-smoothed diagonal Hessian subject to $K$. The final realization of $\left(d c_{m} / d t\right)$ is obtained by a direct model space point-to-point division of the " $M$-smoothed gradient subject to $K$ " by the " $M$ smoothed diagonal Hessian subject to $K$ " followed by another smoothing by $M$. The Euler step (Kincaid and Cheney, 1996) is an update to velocity via $c_{m}(t+\delta t)=c_{m}(t)+\alpha\left(d c_{m} / d t\right)$, where $\alpha$ is taken as a constant throughout the iterations. The implementation of the velocity inversion varies depending on which regularizations are invoked. Example 2 contains no regularization in the image nor in the velocity. Therefore, equation B-1 is implemented directly. Example 3 contains a regularization in the image; thus, equation (21) is implemented. A mild smoothing is applied to the velocity update at the end of each iteration to remove the variability under a length scale of $500 \mathrm{~m}$ in each dimension. In example 4 , regularizations in the image as well as in the velocity are incorporated into the inversion. This requires a full implementation of equation B-5. We report that the scheme described by equa- 
tion B-5 does not rely on heavy smoothing and the implementation of $\mathrm{M}$ can be as simple as smoothing by averaging.

\section{REFERENCES}

Albertin, U., 2011, An improved gradient computation for adjoint waveequation reflection tomography: 81st Annual International Meeting, SEG, Expanded Abstracts, 3969-3973.

Fei, W., and P. Williamson, 2010, On the gradient artifacts in migration velocity analysis based on differential semblance optimization: 80th Annual International Meeting, SEG, Expanded Abstracts, 4071-4076.

Kincaid, D., and W. Cheney, 1996, Numerical analysis: Mathematics of scientific computing: Brooks/Cole Publishing Company.

Olver, P. J., 1993, Applications of Lie groups to differential equations: Springer-Verlag.

Pratt, R. G., C. Shin, and G. J. Hicks, 1998, Gauss-newton and full newton methods in frequency domain seismic waveform inversion: Geophysical Journal International, 133, 341-362, doi: 10.1046/j.1365-246X.1998 $.00498 . x$.
Sava, P., 2000, Prestack Stolt residual migration for migration velocity analysis: 70th Annual International Meeting, SEG, Expanded Abstracts, 992-995.

Shen, P., 2012, An RTM based automatic migration velocity analysis in image domain: 82nd Annual International Meeting, SEG, Expanded Abstracts, doi: 10.1190/segam2012-0214.1.

Shen, P., 2013, Subsurface focusing of diving waves and its application to reflection tomography: 75th Annual International Meeting, EAGE, Extended Abstracts, Th1005.

Shen, P., and W. W. Symes, 2008, Automatic velocity analysis via shot profile migration: Geophysics, 73, no. 5, VE49-VE59, doi: 10.1190/1 .2972021 .

Symes, W. W., 2008, Migration velocity analysis and waveform inversion: Geophysical Prospecting, 56, 765-790, doi: 10.1111/j.1365-2478.2008 .00698.x.

Tarantola, A., and B. Vallette, 1982, Inverse problems: Quest for information: Journal of Geophysics, 50, 159-170.

$\mathrm{Xu}, \mathrm{S}$., H. Chauris, and G. Lambaré, 2001, Common-angle migration: A strategy for imaging complex media: Geophysics, 66, 1877-1894, doi: $10.1190 / 1.1487131$. 